\title{
Light-Emitting Dehalogenases: Reconstruction of Multifunctional Biocatalysts
}

\author{
Radka Chaloupkova, ${ }^{\dagger}$ Veronika Liskova, ${ }^{\dagger}$ Martin Toul, $^{\dagger}$ Klara Markova, $^{\dagger}$ Eva Sebestova, ${ }^{\dagger}$ \\ Lenka Hernychova, ${ }^{\ddagger}$ Martin Marek, ${ }^{\dagger}$ Co Gaspar P. Pinto, ${ }^{\dagger, \|}$ Daniel Pluskal, ${ }^{\dagger}$ Jitka Waterman, ${ }^{\S}$ \\ Zbynek Prokop, ${ }^{\dagger, \| \odot}$ and Jiri Damborsky*,,,$\| \odot$ \\ ${ }^{\dagger}$ Loschmidt Laboratories, Department of Experimental Biology and Research Centre for Toxic Compounds in the Environment \\ RECETOX, Masaryk University, Kamenice 5/A13, 62500 Brno, Czech Republic \\ ${ }^{\ddagger}$ Regional Centre for Applied Molecular Oncology, Masaryk Memorial Cancer Institute, 65653 Brno, Czech Republic \\ ${ }^{\S}$ Diamond Light Source, Harwell Science and Innovation Campus, Didcot OX11 0DE, United Kingdom \\ "International Clinical Research Center, St. Anne’s University Hospital Brno, Pekarska 53, 65691 Brno, Czech Republic
}

Supporting Information

ABSTRACT: To obtain structural insights into the emergence of biological functions from catalytically promiscuous enzymes, we reconstructed an ancestor of catalytically distinct, but evolutionarily related, haloalkane dehalogenases (EC 3.8.1.5) and Renilla luciferase (EC 1.13.12.5). This ancestor has both hydrolase and monooxygenase activities. Its crystal structure solved to $1.39 \AA$ resolution revealed the presence of a catalytic pentad conserved in both dehalogenase and luciferase descendants and a molecular oxygen bound in between two residues typically stabilizing a halogen anion. The differences in the conformational dynamics of the specificity-determining cap domains between the ancestral and descendant enzymes were accessed by molecular dynamics and hydrogen-deuterium exchange mass spectrometry. Stopped-flow analysis revealed that the alkyl enzyme intermediate formed in the luciferase-catalyzed reaction is trapped by blockage of a hydrolytic reaction step. A single-point mutation (Ala54Pro) adjacent to one of the catalytic residues bestowed hydrolase activity on the modern luciferase by enabling cleavage of this intermediate. Thus, a single substitution next to the catalytic pentad may enable the emergence of promiscuous activity at the enzyme class level, and ancestral reconstruction has a clear potential for obtaining multifunctional catalysts.

KEYWORDS: catalytic promiscuity, ancestral reconstruction, haloalkane dehalogenase, monooxygenase, luciferase, emergence of biological function

\section{INTRODUCTION}

Under selective pressures, enzymes have generally developed high catalytic efficiency and specificity. Hence, they are functionally classified in terms of the main substrate conversions they catalyze in vivo (their "native reactions"). However, many have "catalytic promiscuity", i.e., the ability to catalyze reactions involving non-natural substrates or additional reactions. ${ }^{1-4}$ The degree of an enzyme's promiscuity is generally assessed by examining the types of bonds formed or broken during its catalytic activities and differences in mechanisms between native and "promiscuous" reactions. ${ }^{5} \mathrm{~A}$ systematic, hierarchical approach is to classify enzymes' promiscuity in terms of differences in Enzyme Commission (EC) numbers of the reactions they can catalyze. ${ }^{6,7}$ Catalytic promiscuity generally corresponds to the cases in which the EC numbers of the various substrates and reactions catalyzed by the same enzyme differ in the second, or the third, digits that

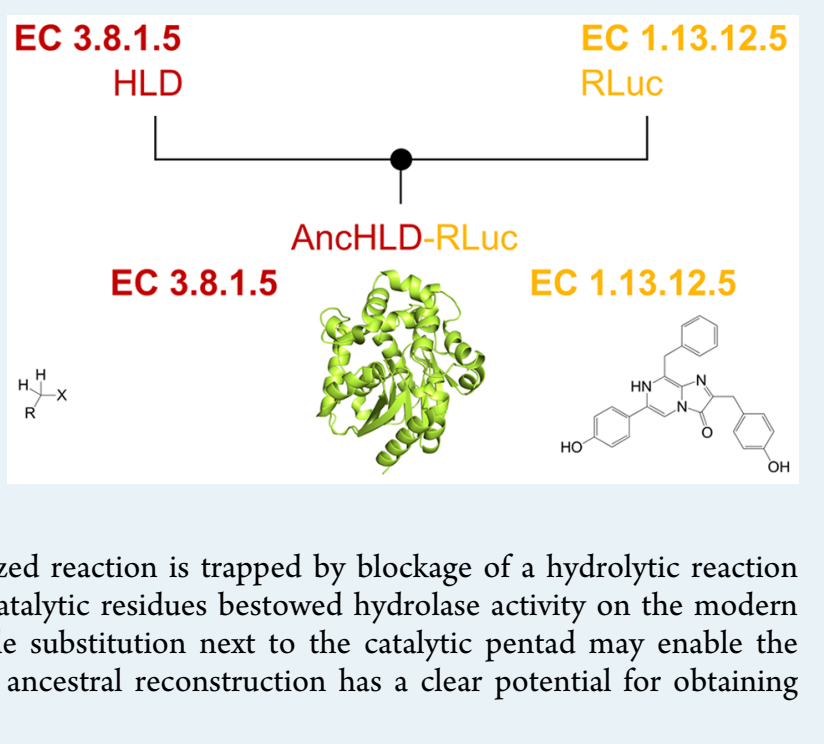

EC 3.8.1.5

HLD

RLuC refer to different chemistries and different classes of substrates or even by the first digit, indicating a completely different reaction category. ${ }^{6}$ The emergence of enzymatic functions has often been associated with the initial occurrence of promiscuity, ${ }^{8}$ and understanding enzyme promiscuity is becoming increasingly important to improve our general knowledge of enzyme evolution and ability to engineer enzymes with unique properties.

Ancestral enzymes putatively had catalytic promiscuity, being "generalists" exhibiting broad substrate specificity and, at the same time, low catalytic efficiency, ${ }^{6,8-11}$ but through mutation, duplication, and horizontal transfer, gene families diversified and variants with more specific and efficient

Received: March 11, 2019

Revised: April 14, 2019

Published: April 15, 2019 

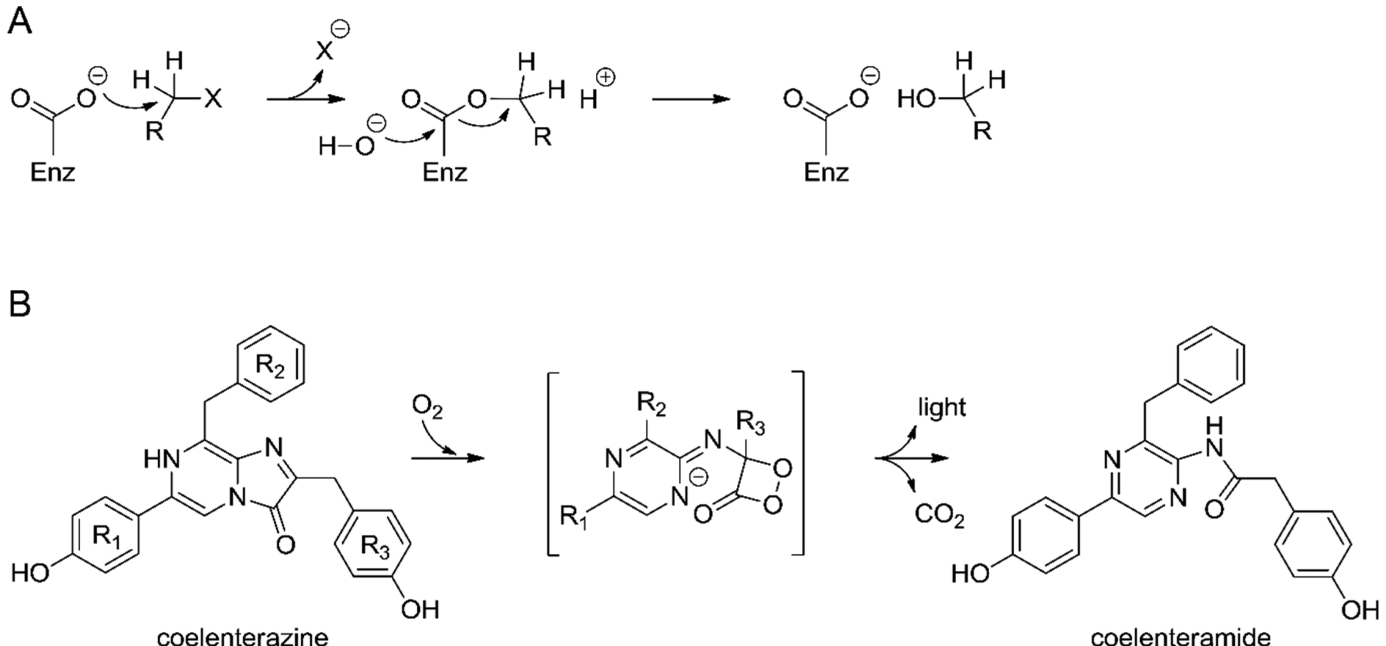

Figure 1. Distinct reaction mechanisms of closely evolutionary related haloalkane dehalogenases (HLDs, EC 3.8.1.5) and a Renilla luciferase (RLuc, EC 1.13.12.5). (A) HLDs catalyze hydrolytic dehalogenation of halogenated alkanes to a primary alcohol, a halide ion $\left(\mathrm{X}^{-}\right)$, and a proton. The two-step mechanism is initiated by a nucleophilic attack of aspartic acid on the carbon atom of the substrate resulting in a covalent alkyl enzyme intermediate. This complex is subsequently hydrolyzed by a water molecule activated by catalytic base. ${ }^{24}$ (B) RLuc catalyzes monooxygenation of coelenterazine in the presence of oxygen, yielding carbon dioxide, blue light, and coelenteramide. The catalytic mechanism of RLuc is poorly understood but is expected to proceed via a dioxetanone intermediate. ${ }^{25,26}$

catalytic activities evolved. ${ }^{6,11-13}$ Although the ancestral enzymes no longer exist, their sequences can be inferred by ancestral sequence reconstruction. ${ }^{4-16}$ Reconstructed ancestral enzymes often exhibit substrate promiscuity. For example, resurrected ancestors of $\alpha$-glucosidases have exhibited hydrolytic activity toward broader ranges of maltose-like and isomaltose-like sugars than their modern descendants. ${ }^{12}$ Similarly, reconstructed ancestors of $\beta$-lactamases, ${ }^{17}$ hydroxymethyl pyrimidine kinases, ${ }^{18}$ carboxyl methyltransferases, ${ }^{19}$ and 2-ketoacid oxidoreductases ${ }^{20,21}$ have all exhibited higher substrate promiscuity than their respective descendants. However, very few examples of resurrected ancestral enzymes exhibiting catalytic promiscuity (i.e., the ability to catalyze different kinds of reactions, rather than the same kind of reaction with similar substrates) have been reported. ${ }^{22,23}$ Moreover, although many reconstructed ancestral hydroxynitrile lyases and esterases at four nodes of a published phylogenetic tree can catalyze both an ester hydrolysis and a cyanohydrin cleavage, the extant enzymes also have some (albeit weaker) promiscuous activity. ${ }^{22}$

In the study reported here, we reconstructed a common ancestor of haloalkane dehalogenases ${ }^{27}$ and Renilla luciferase, ${ }^{28,29}$ which are catalytically specific enzymes although they belong to the same HLD-II enzyme subfamily. ${ }^{30}$ Haloalkane dehalogenases (HLDs, EC 3.8.1.5) catalyze hydrolytic dehalogenation of a broad range of halogenated aliphatic compounds into the corresponding alcohols, a halide anion, and a proton. ${ }^{27,31}$ Luciferase from Renilla reniformis (RLuc, EC 1.13.12.5) 28,29 $^{2}$ catalyzes (with no need for any cofactor) monooxygenation of a coelenterazine, yielding a coelenteramide and a carbon dioxide, with emission of blue light. ${ }^{26,29}$ RLuc is the only characterized monooxygenase, which is clustered in the HLD enzyme family together with recently identified putative luciferase from Amphiura filiformis. ${ }^{32}$ Activity of this putative luciferase with coelenterazine has been detected with the crude extract from arms of the animal, $^{32}$ while production of light by the purified protein needs to be verified. Structurally, both HLDs and RLuc are classified as $\alpha / \beta$-hydrolases with highly conserved catalytic core domain and specificity-determining cap domain. ${ }^{33-35}$ The core domain includes a conserved catalytic pentad composed of a nucleophile, a base, a catalytic acid, and two halidestabilizing residues. ${ }^{27,30}$ However, the reaction mechanism and substrate specificity of HLDs and RLuc are strikingly different (Figure 1). HLDs have a two-step reaction mechanism, involving activated water-mediated hydrolysis of a covalently bound alkyl enzyme intermediate. ${ }^{24,36-40}$ In contrast, the incompletely understood catalytic mechanism of RLuc putatively involves the decomposition of a dioxetanone intermediate that provides energy enabling generation of a product molecule in an electronically excited state. ${ }^{26,25}$ Hydrolytic conversion of halogenated alkanes releases an alcohol, while monooxygenation of coelenterazine results in emission of light. A common ancestor of HLDs and RLuc is an exciting and challenging target for reconstruction, as it could profoundly illuminate elusive aspects of these strongly differing reaction mechanisms. Here, we combined several methods including ancestral sequence reconstruction, X-ray crystallography, steady-state and presteady-state enzyme kinetics, matrixassisted laser desorption ionization-time-of-flight mass spectrometry (MALDI-TOF MS), site-directed mutagenesis, molecular dynamics (MD), and hydrogen-deuterium exchange mass spectrometry (HDX-MS) to get structural insight into the understanding why evolutionary related modern-day enzymes exhibit distinct catalytic functions (Figure S1).

\section{MATERIALS AND METHODS}

Ancestral Sequence Reconstruction. The ancestral sequence was reconstructed as previously described. ${ }^{41}$ Briefly, a nonredundant set of HLD-II protein sequences was collected by PSI-BLAST v2.2.22 $+{ }^{42}$ searches against the NCBI nr database (version 21-9-2012) ${ }^{43}$ and then clustered with CLANS $^{44}$ and CD-HIT v4.5.4. ${ }^{45}$ The data set was divided into two subgroups based on their evolutionary relatedness. For each HLD-II subgroup, a separate multiple sequence alignment (MSA) was constructed by MUSCLE v3.51 $1^{46}$ and refined in BioEdit ${ }^{47}$ based on the manually prepared structureguided MSA. The MSAs of both subgroups were then aligned 
to each other using MUSCLE profile-profile alignment and manually refined. The phylogenetic tree of the HLD-II subfamily was calculated by PhyML v3.0, ${ }^{48}$ based on the LG substitution matrix ${ }^{49}$ combined with $+\mathrm{I},+\mathrm{F}$, and $+\mathrm{G}$ parameters selected by PROTTEST v2.4 ${ }^{50}$ and using the best result from nearest neighbor interchange ${ }^{51}$ and subtree pruning and regrafting ${ }^{52}$ to optimize the tree topology starting from $\mathrm{BIONJ}^{53}$ or five random starting trees, respectively. Confidence levels of the output tree were estimated by bootstrapping the data 100 times. The tree was rooted according to the previously published HLD tree. ${ }^{30}$ For all ancestral nodes of each tree from the obtained distribution of trees, ancestral states at each site of the MSA were predicted by the maximum-likelihood $\operatorname{method}^{54,55}$ using the Codeml module of PAML v4.4 $4^{56}$ and Lazarus software ${ }^{57}$ based on the $\mathrm{LG}+\mathrm{F}+\mathrm{G}$ model. The ancestral state distributions at each site and each node were then obtained by weighting ancestral reconstructions from each tree by the associated posterior probability of a given tree. The most probable ancestor sequence of the selected node was then predicted by assigning to each position the ancestral state with the highest-weighted posterior probability. The ancestral gap characters were initially placed by Lazarus according to Fitch's parsimony ${ }^{58}$ and then refined manually in BioEdit.

Gene Synthesis, Protein Expression, and Purification. Gene encoding inferred sequence of AncHLD-RLuc, LinB, and RLuc8 was synthesized artificially (GeneArt, Life technologies, Regensburg, Germany). The codon usage was automatically adapted to the codon bias of Escherichia coli genes by GeneArt's Web site service. For expression purposes, the ancHLD-RLuc, $\operatorname{lin} B$, and $r L u c 8$ genes were then subcloned into the expression vector pET21b (Novagen, San Diego, USA) between NdeI and BamHI, XhoI, or HindIII restriction sites, respectively. To overproduce AncHLD-RLuc, LinB, and RLuc8 in E. coli, expression of the corresponding genes (under control of the T7lac promoter) was induced by adding isopropyl $\beta$-D-thiogalactopyranoside (IPTG). E. coli strain BL21(DE3) cells containing recombinant plasmids pET21$\mathrm{b}:: a n c H L D-R L u c, p E T 21 \mathrm{~b}:: \operatorname{linB}$ or $\mathrm{pET} 21 \mathrm{~b}::$ Luc8 were grown in 11 of Luria broth medium with ampicillin $\left(100 \mathrm{mg} \mathrm{mL}^{-1}\right)$ at $37^{\circ} \mathrm{C}$. When the culture reached an optical density 0.6 at 600 $\mathrm{nm}$, the enzyme expression (at $20^{\circ} \mathrm{C}$ ) was induced by adding IPTG to a final concentration of $0.5 \mathrm{mM}$. The cells were harvested, disrupted by sonication using an UP200S ultrasonic processor (Hielscher, Teltow, Germany), and centrifuged for 1 $\mathrm{h}$ at $4{ }^{\circ} \mathrm{C}$ and $21000 \mathrm{~g}$. AncHLD-RLuc, LinB, and RLuc8 were purified using a Ni-NTA Superflow Cartridge (Qiagen, Hilden, Germany), as previously described. ${ }^{59}$ Each purified protein was pooled and dialyzed overnight against $50 \mathrm{mM}$ potassium phosphate buffer $\left(\mathrm{pH} \mathrm{7.5)}\right.$ and then stored at $4{ }^{\circ} \mathrm{C}$. The protein concentration was determined using the Bradford reagent (Sigma-Aldrich, St. Louis, MO), and the protein purity was checked by SDS-PAGE. All investigated enzymes were prepared with a purity $\geq 95 \%$ as determined by SDS-PAGE.

Circular Dichroism Spectroscopy and Thermal Denaturation. To assess the secondary structure of the purified enzymes and check that they were correctly folded, circular dichroism (CD) spectra of each enzyme, in $50 \mathrm{mM}$ phosphate buffer $(\mathrm{pH} 7.5)$, were recorded at room temperature using a Chirascan spectropolarimeter (Chirascan, Leatherhead, UK) equipped with a Peltier thermostat. Data were collected from 185 to $260 \mathrm{~nm}$ at a scan rate of $100 \mathrm{~nm} \mathrm{~min}{ }^{-1}$ with a $1 \mathrm{~s}$ response time and a $2 \mathrm{~nm}$ bandwidth using a $0.1 \mathrm{~cm}$ quartz cuvette containing the enzyme. Each spectrum shown represents an average of five individual scans, corrected for absorbance by the buffer. CD data were expressed in terms of the mean residue ellipticity $\left(\Theta_{\text {MRE }}\right)$. Thermal unfolding of the studied enzymes was followed by monitoring the ellipticity at $222 \mathrm{~nm}$ over a temperature range of $20-85{ }^{\circ} \mathrm{C}$ at $0.1 \mathrm{~nm}$ resolution and a heating rate of $1{ }^{\circ} \mathrm{C} \mathrm{min}^{-1}$. The resulting thermal denaturation curves were roughly normalized to represent a signal change between approximately 1 and 0 and were fitted to sigmoidal curves using Origin 8 (OriginLab, MA, USA). Apparent melting temperature $\left(T_{\mathrm{m}, \text { app }}\right)$ was evaluated as the midpoint of the normalized thermal transition.

Specific Activity Measurements. Dehalogenase activities of AncHLD-RLuc, LinB, RLuc8, and RLuc8+Ala54Pro toward 29 halogenated substrates were assayed using a previously described colorimetric method. ${ }^{60}$ Briefly, the release of halide ions was monitored spectrophotometrically at $460 \mathrm{~nm}$ using a SUNRISE microplate reader (Tecan, Grödig/Salzburg, Austria) after reaction with mercuric thiocyanate and ferric ammonium sulfate at $37{ }^{\circ} \mathrm{C}$ in $25 \mathrm{~mL}$ Reacti-flasks with Mininert valves. Each reaction mixture contained $10 \mathrm{~mL}$ of glycine buffer (100 mM, pH 8.6) and $10 \mathrm{~mL}$ of a selected halogenated substrate at a concentration of $0.1-10 \mathrm{mM}$ depending on its solubility. The reaction was initiated by adding enzyme and monitored by withdrawing $1 \mathrm{~mL}$ samples periodically from the reaction mixture then immediately mixing them with $0.1 \mathrm{~mL}$ of $35 \%$ nitric acid to terminate the reaction. Dehalogenation activity was quantified as the rate of product formation with time. The limit of detection for dehalogenase activity by the Iwasaki assay with chlorinated, brominated, and iodinated substrates is $0.0030,0.0006$, and $0.0007 \mathrm{nmol} \mathrm{s}^{-1} \mathrm{mg}^{-1}$, respectively.

Monooxygenase activities of AncHLD-RLuc, LinB, RLuc8, and RLuc8+Ala54Pro with coelenterazine were determined using a FLUOStar OPTIMA luminometer (BMG Labtech, Ortenberg, Germany) and expressed in relative light units (RLU) $\mathrm{s}^{-1} \mathrm{mg}^{-1}$ of an enzyme. Samples of $25 \mu \mathrm{L}$ of purified enzyme solution ( $1 \mathrm{mg} \mathrm{mL}^{-1}$ of AncHLD-RLuc, $0.2 \mu \mathrm{g} \mathrm{mL}^{-1}$ of RLuc8, or $2.0 \mu \mathrm{g} \mathrm{mL}^{-1}$ of RLuc8+Ala54Pro) were placed in microplate wells. After baseline collection for $10 \mathrm{~s}$, the luminescence reaction was initiated by adding $225 \mu \mathrm{L}$ of 8.8 $\mu \mathrm{M}$ coelenterazine in reaction buffer $(100 \mathrm{mM}$ potassium phosphate buffer, $\mathrm{pH} 7.5$ ). The RLUs were integrated over the first $72.5 \mathrm{~s}$ immediately after injection of the substrate into the enzyme solution. The activity of each enzyme sample was measured in at least three independent experiments.

Steady-State Kinetic Measurements. A VP-ITC isothermal titration microcalorimeter (MicroCal, Piscataway, USA) was used to characterize steady-state kinetics (at 37 ${ }^{\circ} \mathrm{C}$ ) of conversions of: 1-bromobutane, 1-iodopropane, 1,2dibromoethane, 1,3-dibromopropane, 4-butyronitrile, and 1,2,3-tribromopropane catalyzed by AncHLD-RLuc; 1-iodopropane, 4-butyronitrile, and 1,2,3-tribromopropane catalyzed by LinB; and 1,2-dibromoethane catalyzed by RLuc8+Ala54Pro. In each case, the enzyme was dialyzed overnight against 100 $\mathrm{mM}$ glycine buffer, $\mathrm{pH}$ 8.6. For most of the experiments, 1.4 $\mathrm{mL}$ of enzyme solution at a concentration of $0.003-0.4 \mathrm{mg}$ $\mathrm{mL}^{-1}$ was placed in a reaction cell. The substrate solution was prepared in the same buffer, and its concentration was verified using a gas chromatograph supplied by Finnegan (San Jose, USA). The enzyme was titrated at $150 \mathrm{~s}$ intervals in the reaction cell with increasing amounts of the substrate, while pseudo-first-order conditions were maintained. Every injection 
increased the substrate concentration, leading to a further increase in the enzyme reaction rate (and hence an increase in generated heat) until the enzymatic reaction was saturated. A total of 28 injections were carried out during titration. The reaction rates recorded after every injection (in units of thermal power) were converted to enzyme turnover. Plots of calculated enzyme turnover versus substrate concentration after every injection were then fitted by nonlinear regression to kinetic models using Origin 8 (OriginLab, Northampton, MA). The only exception to this procedure was that to characterize kinetics of the 1,2,3-tribromopropane conversion catalyzed by LinB, $1.4 \mathrm{~mL}$ of a substrate solution in $100 \mathrm{mM}$ glycine buffer $\mathrm{pH} 8.6$ was placed in the microcalorimeter's reaction cell, and the reaction was initiated by injecting $10 \mathrm{~mL}$ of enzyme solution $\left(0.4 \mathrm{mg} \mathrm{mL}^{-1}\right)$ into the cell. The measured rate of heat change was assumed to be directly proportional to the velocity of the enzymatic reaction according to

$$
\frac{\mathrm{d} Q}{\mathrm{~d} t}=-\Delta H V \frac{\mathrm{d}[\mathrm{S}]}{\mathrm{d} t}
$$

where $\Delta H$ is the enthalpy of the reaction, $[\mathrm{S}]$ is the substrate concentration, and $V$ is the volume of the cell. $\Delta H$ was determined by titrating the substrate into the reaction cell containing the enzyme. The reaction was allowed to proceed to completion. The integrated total heat of reaction was divided by the amount of injected substrate. The evaluated rate of substrate depletion $(-\mathrm{d}[S] / \mathrm{d} t)$, and corresponding substrate concentrations were then fitted by nonlinear regression to kinetic models using Origin 8 (OriginLab, Northampton, MA).

Steady-state kinetics of the coelenterazine conversion catalyzed by AncHLD-RLuc, RLuc8 and RLuc8+Ala54Pro (at $37{ }^{\circ} \mathrm{C}$ in wells of the microtiter plates) were evaluated by monitoring the luminescence generated using a FLUOStar OPTIMA luminometer (BMG Labtech, Ortenberg, Germany). Initially, $25 \mu \mathrm{L}$ of purified enzyme solutions $\left(1 \mathrm{mg} \mathrm{mL}^{-1}\right.$ of AncHLD-RLuc, $0.2 \mu \mathrm{g} \mathrm{mL}{ }^{-1}$ of RLuc8, or $2.0 \mu \mathrm{g} \mathrm{mL}^{-1}$ of RLuc8+Ala54Pro) were placed in the wells. Then, after baseline collection for $10 \mathrm{~s}, 225 \mu \mathrm{L}$ of $100 \mathrm{mM}$ potassium phosphate buffer, $\mathrm{pH} 7.5$, containing the substrate at particular concentration ranging from 0.022 to $8.8 \mu \mathrm{M}$ was added. The RLUs were integrated over the first $72.5 \mathrm{~s}$ immediately after injection of the substrate into the enzyme solution. The activity of each enzyme sample was measured in at least three independent experiments. Plots of determined luminescence activity, in $\mathrm{RLUs}^{-1} \mathrm{mg}^{-1}$, versus coelenterazine concentration were fitted by nonlinear regression to kinetic models using Origin 8 (OriginLab, Northampton, MA).

Crystallization and Data Collection. Purified AncHLDRLuc enzyme, concentrated to $10-15 \mathrm{mg} \mathrm{mL}^{-1}$ and stored in $50 \mathrm{mM}$ Tris- $\mathrm{HCl}$ buffer ( $\mathrm{pH} 7.5$ ) was used for crystallization screening. Crystals suitable for X-ray diffraction analysis were obtained from a JCSG-plus screen (Molecular Dimensions, Newmarket, UK) at $292 \mathrm{~K}$ using the sitting-drop vapordiffusion method with a protein/precipitant ratio of $1: 1$. The crystallization solution consisted of $0.1 \mathrm{M}$ HEPES $\mathrm{pH} 7.0$ and $10 \%(w / v)$ PEG 6000. Single crystals of AncHLD-RLuc with average dimensions of $70.6 \times 39.7 \times 8.8 \mu \mathrm{m}$ were cryoprotected by soaking them in a drop containing the crystallization solution supplemented with $25 \%$ (v/v) 2methyl-2,4-pentanediol (MPD) for $5 \mathrm{~s}$. The crystals were then mounted in a SPINE cryoloops (MiTeGen, Ithaca, USA) and flash-cooled in liquid-nitrogen. Diffraction data were collected at the Diamond Light Source beamline I02 to $1.39 \AA$ resolution at $0.9795 \AA$ wavelength using a Pilatus $6 \mathrm{M}-\mathrm{F}$ detector. In total, 1200 images were collected at $100 \mathrm{~K}$ with an oscillation range of $0.15^{\circ}$ per image. The data were processed with Mosflm v7.1.0 ${ }^{61}$ using the first 900 images for indexing and integration, and Aimless v0.2.17 $7^{62}$ for scaling and merging. The crystal belongs to primitive monoclinic space group $P 12_{1} 1$ with unit-cell parameters $a=44.549, b=60.51, c=123.63 \AA$, $\alpha$ $=\gamma=90^{\circ}, \beta=90.9^{\circ}$. The Matthews coefficient $\left(V_{\mathrm{M}}\right)^{63,64}$ of $2.32 \AA^{3} \mathrm{Da}^{1-}$ shows that the crystal contains two molecules per asymmetric unit with estimated solvent content $46.93 \%$.

Structure Determination and Refinement. The structure of AncHLD-RLuc was solved by molecular replacement using Phaser software ${ }^{65}$ implemented in the CCP4 package $^{66}$ and the structure of RLuc8 from $R$. reniformis (PDB ID $2 \mathrm{PSF})^{67}$ as a search model. Manual model building was performed in Coot v0.8.1 ${ }^{68}$ using $2 F_{\mathrm{o}}-F_{\mathrm{c}}$ and $F_{\mathrm{o}}-F_{\mathrm{c}}$ maps, followed by several cycles of automated refinement with REFMAC $v 5.8^{69}$ from the CCP4 package ${ }^{66}$ and phenix.refine from the Phenix package ${ }^{70}$ using the anisotropic B-factor model. The final refinement step and model optimization was performed using PDB REDO. ${ }^{71}$ The refinement statistics are summarized in Table S1. Figures showing structural representations were prepared using PyMOL v1.5.0.4. ${ }^{72}$ Atomic coordinates and experimental structure factors have been deposited in the Protein Data Bank under PDB code $6 \mathrm{G} 75$.

HDX-MS Analysis. Deuteration of AncHLD-RLuc, RLuc8, and LinB was followed with the peptides generated after pepsin and nepenthesin digestion. The enzymes $(2 \mu \mathrm{M}$ final concentration) were diluted either with $50 \mu \mathrm{L}$ of $100 \mathrm{mM}$ phosphate buffer in $\mathrm{H}_{2} \mathrm{O}(\mathrm{pH}$ 7.5) and used for the preparation of undeuterated control and for the peptide mapping or with $100 \mathrm{mM}$ phosphate buffer in $\mathrm{D}_{2} \mathrm{O}(\mathrm{pD} 7.1$ ) and used for the preparation of the deuterated samples. The hydrogen-deuterium exchange was carried out at room temperature and was quenched at 10 and $60 \mathrm{~s}$ by the addition of $1 \mathrm{M} \mathrm{HCl}$ in $1 \mathrm{M}$ glycine with pepsin. Each sample was directly injected into LC-system (UltiMate 3000 RSLCnano, Thermo Fisher Scientific, Waltham, MA) to an immobilized nepenthesin enzymatic column $(15 \mu \mathrm{L}$ bed volume, flow rate $20 \mu \mathrm{L} / \mathrm{min}, 2 \%$ acetonitrile $/ 0.05 \%$ trifluoroacetic acid). Peptides were trapped and desalted online using a peptide microtrap (Michrom Bioresources, Auburn, CA) for $3 \mathrm{~min}$ at the flow rate $20 \mu \mathrm{L} / \mathrm{min}$. Then the peptides were eluted onto an analytical column (Jupiter C18, $1.0 \times 50 \mathrm{~mm}, 5 \mu \mathrm{m}, 300 \AA$, Phenomenex, CA) and separated using 2 min linear gradient elution of $10-40 \%$ of buffer B ( $80 \%$ acetonitrile/ $0.08 \%$ formic acid) in buffer $\mathrm{A}$ ( $0.1 \%$ formic acid), followed by $31 \mathrm{~min}$ isocratic elution at $40 \%$ of buffer B. The immobilized nepenthesin column, trap cartridge, and the analytical column were kept at $1{ }^{\circ} \mathrm{C}$. The mass spectrometric analysis was carried out using the Orbitrap Elite mass spectrometer (Thermo Fisher Scientific, Waltham, MA) with ESI ionization connected with a robotic system based on the HTS-XT platform (CTC Analytics AG, Zwingen, Switzerland). The instrument was operated in a data-dependent mode suitable for peptide mapping (HPLC-MS/MS). Each MS scan was followed by MS/MS scans of the top three most intensive ions from both CID and HCD fragmentation spectra. Tandem mass spectra were searched using the SequestHT against the cRap protein database (ftp://ftp.thegpm.org/fasta/cRAP) containing sequences of tested enzymes. Sequence coverage was analyzed with the Proteome Discoverer 1.4 software (Thermo Fisher 
Scientific, Waltham, MA). Analysis of deuterated samples was done in LC-MS mode with ion detection in the orbital ion trap and the data were processed using HDX Examiner vision 2.2 (Sierra Analytics, Modesto, CA).

MD Simulations. The crystal structures of $\mathrm{LinB}^{73}$ (PDB ID 1MJ5) and RLuc8 ${ }^{67}$ (PDB ID 2PSF, chain B) were retrieved from the Protein Data Bank. The crystal structure of the AncHLD-RLuc (PDB ID 6G75) was obtained as described in the previous sections. The $\mathrm{H}++$ web server ${ }^{74}$ was used to add the missing hydrogens, neutralize, and create a water box with an edge distance of $10 \AA$. All three systems were equilibrated using the Equilibration_v2 module of HTMD. ${ }^{75}$ The systems were first minimized using the conjugate-gradient method for 500 steps. Next, the systems were heated and minimized as follows: (i) 500 steps ( 2 ps) of NVT heating, with the Berendsen barostat, to $310 \mathrm{~K}$, with constraints on all the protein heavy atoms; (ii) $2.5 \mathrm{~ns}$ of NPT equilibration, with the Langevin thermostat, with $1 \mathrm{kcal} \mathrm{mol}^{-1} \AA^{-2}$ constraints on all the protein heavy atoms and (iii) $2.5 \mathrm{~ns}$ of NPT equilibration, with the Langevin thermostat without constraints. During the equilibration simulations, holonomic constraints were applied on all hydrogen-heavy atom bond terms and the mass of hydrogen atoms was scaled with a factor of 4 , enabling the 4 fs time step. ${ }^{76-79}$ The simulations employed periodic boundary conditions, using the particle mesh Ewald method for the treatment of interactions beyond a $9 \AA$ cutoff, electrostatic interactions were suppressed $>4$ bond terms away from each other and the smoothing and switching of van der Waals and electrostatic interactions cutoff at $7.5 \AA^{76}$ HTMD was used to perform adaptive sampling of the RMSD of the $\mathrm{C} \alpha$ atoms of residues 10 to 290 . The $20 \mathrm{~ns}$ production runs were started with the files resulting from the equilibration and the same settings as the last step of the equilibration were used. The trajectories were saved every 100 ps. Total simulation times were higher than $4 \mu$ s for all three systems. The data were clustered using MiniBatchKmeans algorithm to 200 clusters. A lag time of $15 \mathrm{~ns}$ was used to construct the 2 Markov states (open and closed). The final data was calculated from 1000 bootstrapping runs of $50 \%$ of the data.

Analysis of Protein Tunnels. The access tunnels were calculated by the Caver 3.02 for 1000 random frames belonging to each state using the Caver Analyst 2.0.31, with the probe size: $0.9 \AA$, clustering threshold: 5 , shell depth: $5 \AA$, and shell radius: $5 \AA^{80}$ The starting point was defined by five analogous catalytic residues located inside the active site cavity. For AncHLD-RLuc, these residues are the nucleophile Asp118, the catalytic base His284, the catalytic acid Glu142, and two halide/oxygen-stabilizing residues Asn51 and Trp119. For RLuc8, these residues are the nucleophile Asp120, the catalytic base His285, the catalytic acid Glu132, and two halide/oxygenstabilizing residues Asn53 and Trp121. For LinB, these residues are the nucleophile Asp108, the catalytic base His272, the catalytic acid Glu142, and two halide-stabilizing residues Asn38 and Trp109.

Site-Directed Mutagenesis. The single-point variant of RLuc8 (RLuc8+Ala54Pro) was constructed by site-directed mutagenesis using a set of mutagenic oligonucleotides (Merck, Darmstadt, Germany): 5' TTTCTGCATGGTAATCCGACCAGCAGCTATCTG $3^{\prime}$ (forward) and $5^{\prime}$ CAGATAGCTGCTGGTCGGATTACCATGCAGAAA 3' (reverse) with pET21b:: $r L u c 8$ as a template. The mutagenesis involved twostep PCR, with $50 \mu \mathrm{L}$ reaction mixtures containing $100 \mathrm{ng}$ of template DNA, $10 \mathrm{pmol}$ of each oligonucleotide, $0.02 \mu \mathrm{M}$ of each dNTP (New England Biolabs, Ipswich, MA), and 2.5 U of Phusion HF DNA Polymerase in Phusion HF buffer with $1.5 \mathrm{mM} \mathrm{MgCl} 2$ (New England Biolabs). In the first step, a linear product with the desired mutation was synthesized using $\mathrm{Fw}$ and $\mathrm{Rv}$ oligonucleotides. The product served as a megaprimer in the second round of PCR where the whole plasmid was synthesized. The temperature program for the first round consisted of $30 \mathrm{~s}$ at $95^{\circ} \mathrm{C}$, followed by nine cycles of $8 \mathrm{~s}$ at $98{ }^{\circ} \mathrm{C}, 20 \mathrm{~s}$ at $55^{\circ} \mathrm{C}$ and $143 \mathrm{~s}$ at $72{ }^{\circ} \mathrm{C}$. The second round involved 19 cycles of $8 \mathrm{~s}$ at $98{ }^{\circ} \mathrm{C}, 20 \mathrm{~s}$ at $55^{\circ} \mathrm{C}$, and $150 \mathrm{~s}$ at $72{ }^{\circ} \mathrm{C}$ followed by $10 \mathrm{~min}$ at $72{ }^{\circ} \mathrm{C}$. PCR products were treated with the methylation-dependent endonuclease DpnI (New England Biolabs) for $1 \mathrm{~h}$ at $37{ }^{\circ} \mathrm{C}$. The resulting plasmids were transformed by a heat shock method into E. coli Dh5 $\alpha$ cells (ZymoResearch, Orange, CA) and amplified. The presence of desired mutations was confirmed by sequence analyses at GATC Biotech (Constance, Germany).

Stopped-Flow Experiments. Stopped-flow fluorescence single-turnover experiments were performed using an SFM3000 stopped-flow instrument combined with a MOS-500 spectrometer (Bio-Logic Science Instruments, SeyssinetPariset, France). Fluorescence emissions from tryptophan residues of each enzyme included in the analyses were observed through a $320 \mathrm{~nm}$ cutoff filter upon excitation at $295 \mathrm{~nm}$. Single turnover fluorescence traces were recorded after rapidly mixing $75 \mu \mathrm{L}$ of AncHLD-RLuc $(75 \mu \mathrm{M})$, RLuc8 $(209 \mu \mathrm{M})$, RLuc8+Ala54Pro $(99 \mu \mathrm{M})$, or LinB $(124 \mu \mathrm{M})$ solutions with $75 \mu \mathrm{L}$ of 1-bromobutane in a concentration $12.7,34.5,21.4$, or $40.9 \mu \mathrm{M}$, respectively. Repeating singleturnover fluorescence experiments were performed using a Synergy H4 Hybrid Microplate Reader (BioTek, Winooski, $\mathrm{VT})$. In the reaction with 1 -bromobutane, $200 \mu \mathrm{L}$ of RLuc8 $(89 \mu \mathrm{M})$ or RLuc8+Ala54Pro $(137 \mu \mathrm{M})$ solutions was injected into three independent wells and mixed with $20 \mu \mathrm{L}$ of the substrate solution in a concentration 85.4 or $52.7 \mu \mathrm{M}$, respectively. In the reaction with 1,2-dibromoethane, $200 \mu \mathrm{L}$ of RLuc8 $(89 \mu \mathrm{M})$ or RLuc8+Ala54Pro $(767 \mu \mathrm{M})$ solutions was injected into three independent wells and mixed with 20 $\mu \mathrm{L}$ of the substrate solution in a concentration 116.8 or 747.1 $\mu \mathrm{M}$, respectively. The reaction was monitored through timedependent changes in intrinsic fluorescence of the enzyme at $340 \mathrm{~nm}$ upon excitation at $295 \mathrm{~nm}$. When the single turnover reaction was completed, two further $20 \mu \mathrm{L}$ portions of the substrate solution were injected into each well containing the enzyme to monitor second and third turnovers of the enzyme catalysis. In each case, the solutions were prepared in $100 \mathrm{mM}$ glycine buffer ( $\mathrm{pH} 8.6)$ and incubated at $37^{\circ} \mathrm{C}$.

MALDI-TOF MS Analysis. Purified RLuc8 and RLuc8+Ala54Pro were dialyzed overnight against $100 \mathrm{mM}$ glycine buffer, $\mathrm{pH}$ 8.6, before analysis. The enzyme-substrate reaction proceeded at $37{ }^{\circ} \mathrm{C}$ in a $0.5 \mathrm{~mL}$ reaction mixture with $1-15$ $\mathrm{mg} \mathrm{mL} \mathrm{m}^{-1}$ of the enzyme and 3- to 30 -fold excess of the substrate (1-bromobutane). The reaction was initiated by mixing the enzyme in the reaction buffer with the substrate and monitored by withdrawing samples periodically from the reaction mixture and immediately mixing them with MALDI matrix to terminate the reaction. MALDI-TOF mass spectra were recorded using an Ultraflextreme instrument (Bruker Daltonics, Bremen, Germany) operated in linear mode with detection of positively charged ions. Ferulic acid $(12.5 \mathrm{mg}$ $\mathrm{mL}^{-1}$ in a water-acetonitrile-formic acid mixture, 50:33:17, $\mathrm{v} / \mathrm{v} / \mathrm{v})$ was used as the MALDI matrix; $0.6 \mu \mathrm{L}$ of each reaction sample was mixed with $2.4 \mu \mathrm{L}$ of matrix solution, and then 0.6 
Table 1. Steady-State Kinetic Parameters for Hydrolytic Dehalogenation of Selected Halogenated Substrates Catalyzed by AncHLD-RLuc, ${ }^{a}$ LinB, ${ }^{a}$ and RLuc8+Ala54Pro ${ }^{a}$ and Monooxygenation of Coelenterazine Catalyzed by AncHLD-RLuc, RLuc8, and RLuc8+Ala54Pro ${ }^{a}$

\begin{tabular}{|c|c|c|c|}
\hline & \multicolumn{3}{|c|}{ dehalogenase activity } \\
\hline & $k_{\text {cat }}\left(\mathrm{s}^{-1}\right)$ & $K_{\mathrm{m}}(\mathrm{mM})$ & $k_{\text {cat }} / K_{\mathrm{m}}\left(\mathrm{mM}^{-1} \mathrm{~s}^{-1}\right)$ \\
\hline \multicolumn{4}{|l|}{ AncHLD-RLuc } \\
\hline 1-bromobutane & $0.55 \pm 0.03$ & $0.043 \pm 0.002$ & $12.8 \pm 0.9$ \\
\hline 1-iodopropane & $0.82 \pm 0.07$ & $0.15 \pm 0.02$ & $5.47 \pm 0.88$ \\
\hline 1,2-dibromoethane & $0.290 \pm 0.004$ & $1.72 \pm 0.04$ & $0.169 \pm 0.005$ \\
\hline 1,3-dibromopropane & $1.6 \pm 0.3$ & $0.07 \pm 0.01$ & $22.5 \pm 4.9$ \\
\hline 4-butyronitrile & $1.0 \pm 0.2$ & $0.18 \pm 0.06$ & $5.50 \pm 2.32$ \\
\hline 1,2,3-tribromopropane & $0.034 \pm 0.009$ & $0.039 \pm 0.007$ & $0.872 \pm 0.264$ \\
\hline \multicolumn{4}{|l|}{$\operatorname{Lin} B$} \\
\hline 1-bromobutane & $2.26 \pm 0.07^{b}$ & $0.121 \pm 0.002^{b}$ & $18.7 \pm 0.7^{b}$ \\
\hline 1-iodopropane & $2.05 \pm 0.05$ & $0.041 \pm 0.002$ & $50.0 \pm 2.5$ \\
\hline 1,2-dibromoethane & $12 \pm 4^{c}$ & $1.7 \pm 0.2^{c}$ & $7.11 \pm 2.47^{c}$ \\
\hline 1,3-dibromopropane & $41 \pm 5^{b}$ & $24 \pm 3^{b}$ & $1.70 \pm 0.31^{b}$ \\
\hline 4-butyronitrile & $5.03 \pm 0.06$ & $0.31 \pm 0.02$ & $16.2 \pm 1.1$ \\
\hline 1,2,3-tribromopropane & $4.0 \pm 0.1$ & $0.59 \pm 0.05$ & $6.83 \pm 0.61$ \\
\hline \multicolumn{4}{|l|}{ RLuc8+Ala54Pro } \\
\hline \multirow[t]{3}{*}{ 1,2-dibromoethane } & $0.052 \pm 0.002$ & $1.2 \pm 0.2$ & $0.043 \pm 0.007$ \\
\hline & \multicolumn{3}{|c|}{ monooxygenase activity } \\
\hline & $V_{\lim }\left(\mathrm{RLU} \mathrm{s}^{-1} \mathrm{mg}^{-1}\right)$ & $K_{\mathrm{m}}(\mu \mathrm{M})$ & $V_{\lim } / K_{\mathrm{m}}\left(\mu \mathrm{M}^{-1} \mathrm{RLU} \mathrm{s}^{-1} \mathrm{mg}^{-1}\right)$ \\
\hline $\begin{array}{l}\text { AncHLD-RLuc } \\
\text { coelenterazine } \\
\text { RLuc8 }\end{array}$ & $73 \pm 2$ & $1.3 \pm 0.1$ & $53.8 \pm 0.09$ \\
\hline coelenterazine & $(1.63 \pm 0.06) \times 10^{7}$ & $1.1 \pm 0.1$ & $(1.4 \pm 0.2) \times 10^{7}$ \\
\hline $\begin{array}{l}\text { RLuc8+Ala54Pro } \\
\text { coelenterazine }\end{array}$ & $(4.9 \pm 0.6) \times 10^{6}$ & $5.4 \pm 0.9$ & $(9 \pm 2) \times 10^{5}$ \\
\hline
\end{tabular}

${ }^{a}$ For simplicity, only turnover number $\left(k_{\text {cat }}\right)$, Michaelis constant $\left(K_{\mathrm{m}}\right)$, and catalytic efficiency $\left(k_{\mathrm{cat}} / K_{\mathrm{m}}\right)$ of AncHLD-RLuc, LinB, and RLuc8+Ala54Pro toward tested halogenated substrates are presented in the table. All kinetic parameters determined for the reactions of AncHLDRLuc and LinB with the halogenated substrates are presented in Table S3. All kinetic parameters determined for the reactions of RLuc8+Ala54Pro with 1,2-dibromoethane and coelenterazine are presented in Table S4. The limiting value of maximal velocity $\left(V_{\text {lim }}\right)$ represents 72.5 -s bioluminescence intensity readings standardized for protein content and expressed as RLU s${ }^{-1} \mathrm{mg}^{-1}$ of an enzyme. RLU stands for relative light units. ${ }^{b}$ Data from Chaloupkova et al. ${ }^{59}$ Data from Brezovsky et al. ${ }^{87}$ The standard deviation values are presented.

$\mu \mathrm{L}$ of the mixture was placed on a stainless steel MALDI target. Protein Calibration Mixture II (Bruker Daltonics) was used for external calibration of the mass spectra, which were processed with FlexAnalysis 3.4 software (Bruker Daltonics).

\section{RESULTS}

Catalytic Promiscuity of the Reconstructed Common Ancestor of Haloalkane Dehalogenases and Renilla Luciferase. Ancestral sequence reconstruction ${ }^{14-16}$ is a very powerful method that can provide important insights into the underlying determinants of protein structure, function, and evolution. We have recently inferred the sequences of five highly stable ancestral enzymes corresponding to the different nodes of the HLD-II phylogenetic tree. ${ }^{41}$ Here, we report construction and detailed biochemical characterization of the common ancestor of HLDs and RLuc, denoted AncHLDRLuc. The ancestral state distributions at each site of the AncHLD-RLuc node were predicted by the maximum likelihood method using 93 sequences available for the HLD-II subfamily (Figure S2). The most probable sequence of AncHLD-RLuc was inferred by assigning the ancestral state with the highest-weighted posterior probability to each site (Figures S3 and S4), using the previously established workflow. ${ }^{41}$ Biochemical properties of AncHLD-RLuc were compared with those of modern-day enzymes RLuc8 ${ }^{81}$ (luciferase from $R$. reniformis stabilized by eight single-point mutations necessary for determination of its crystal structure $\left.{ }^{67}\right)$ and $\operatorname{LinB}^{82}$ (1,3,4,6-tetrachloro-1,4-cyclohexadiene hydrolase from Sphingobium japonicum UT26, one of two members of HLD-II subfamily with known catalytic mechanism, ${ }^{44}$ tertiary structure, ${ }^{73,83}$ and biological function ${ }^{84}$ ). Compared to the natural enzyme, RLuc8 exhibits 10 times better yield, 4 times higher monooxygenase activity, 200 times higher resistance to murine serum inactivation, and a small 5 $\mathrm{nm}$ red shift in the emission spectrum. ${ }^{67,81}$ The closest characterized modern-day enzyme is $\mathrm{DspA} ;{ }^{85}$ however, nothing is known about its structure, kinetic mechanism, and biological role. The AncHLD-RLuc sequence has 48\%, 56\%, and $64 \%$ identity with sequences of the modern-day enzymes LinB, ${ }^{82} \mathrm{RLuc}_{8}{ }^{81}$ and DspA, ${ }^{85}$ respectively. The predicted sequence of AncHLD-RLuc contains the catalytic pentad AspHis-Glu + Trp-Asn of modern descendants, and it was unclear whether it would exhibit dehalogenase or monooxygenase activity.

The gene encoding the inferred ancestral sequence was synthesized, and the protein was overproduced in E. coli strain BL21(DE3) with an average yield of $250 \mathrm{mg}$ of soluble purified protein per liter of cell culture. As shown in Figure S5, AncHLD-RLuc exhibited correct folding and greater thermal stability than LinB and RLuc8 (23.4 and $7.8{ }^{\circ} \mathrm{C}$ higher apparent melting points, respectively). We tested HLD activities of AncHLD-RLuc and LinB toward a set of 29 
A

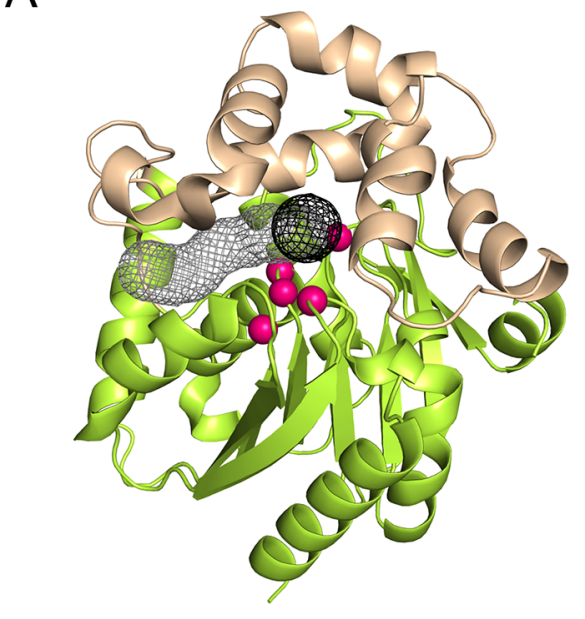

C

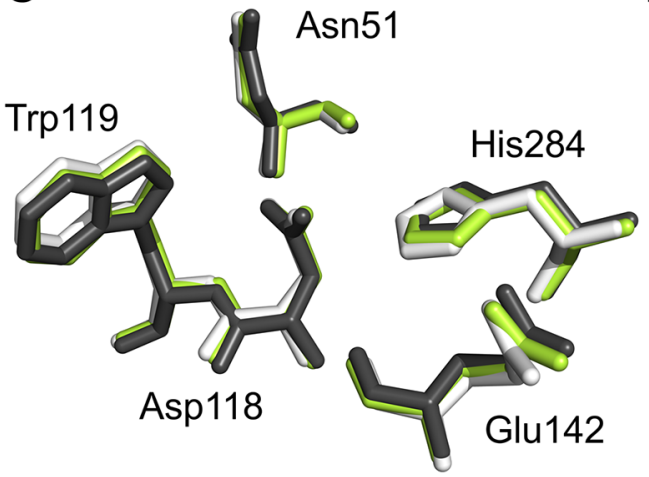

B

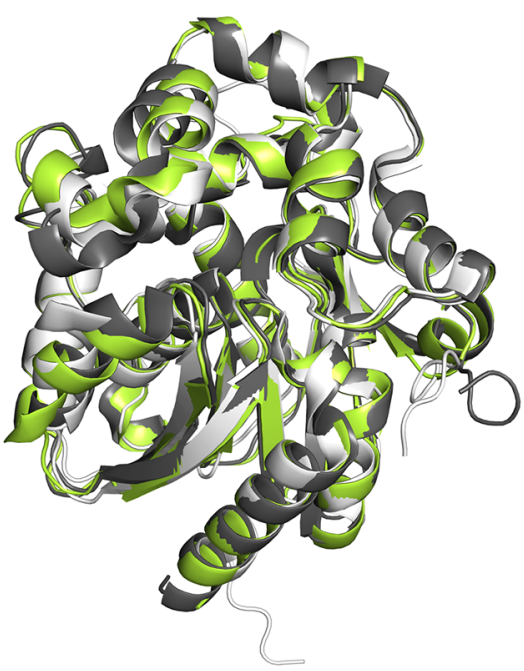

D

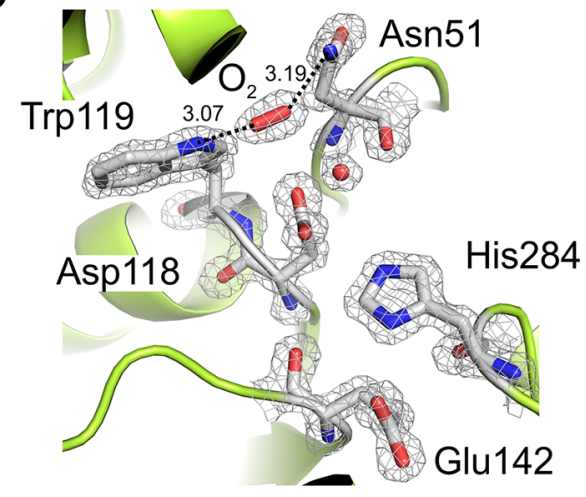

Figure 2. Structural analysis of AncHLD-RLuc. (A) Overall structure of AncHLD-RLuc shown in cartoon representation. The main domain is shown in green; the cap domain is in beige; the catalytic pentad residues are shown as pink balls; the main and the slot tunnels connecting the buried active site with the protein surface are shown as black and gray mesh. (B) Superimposition of crystal structures of AncHLD-RLuc, LinB, and RLuc8. (C) Superimposition of the catalytic pentad residues of AncHLD-RLuc, LinB, and RLuc8. Crystal structures of AncHLD-RLuc, LinB, and RLuc8 are shown in green, white, and black, respectively. (D) Expanded view of the AncHLD-RLuc active site. $2 F_{\mathrm{o}}-F_{\mathrm{c}}$ electron density map contoured at $2.0 \sigma$ is shown for the active site residues with bound $\mathrm{O}_{2}$ ligand. The ligand and interacting residues are displayed in stick representation and the water molecule in sphere representation. Distances between the molecular oxygen and the halide/oxygen stabilizing residues Asn51 and Trp119 are indicated by the numbers (in $\AA$ ) over the black dashed lines.

halogenated compounds. ${ }^{86}$ The ancestral enzyme exhibited lower activity than LinB toward 25 of these 29 compounds, by factors of 2 to 30 (Table S2). AncHLD-RLuc's dehalogenase activity was highest with terminally substituted brominated and iodinated propanes and butanes (Table S2).

Interestingly, AncHLD-RLuc exhibited activity toward several recalcitrant compounds (such as 1,2-dichloroethane, 1,2-dichloropropane, and 1,2-dibromo-3-chloropropane) that most HLDs, including LinB, have no activity toward. ${ }^{86}$ No dehalogenating activity of RLuc8 was detected with any of the tested halogenated substrates (Table S2). Monooxygenase activity of AncHLD-RLuc was tested with coelenterazine, the natural substrate of RLuc. Strikingly, the ancestral enzyme also exhibited clearly detectable monooxygenase activity (Table S2), but much lower ( $>200000$ times) activity than the modern enzyme (ca. $0.0004 \%$ activity of RLuc8). LinB shows no detectable activity toward coelenterazine (Table S2). Thus, AncHLD-RLuc can clearly catalyze both hydrolytic conversion of halogenated substrates and monooxygenation of coelenterazine.
Steady-State Kinetics of AncHLD-RLuc. The catalytic properties of AncHLD-RLuc were assessed by measuring its steady-state kinetic parameters for reactions with six halogenated substrates and coelenterazine and then comparing them to those of $\operatorname{LinB}$ and RLuc8 (Tables 1 and S3). AncHLD-RLuc exhibited lower catalytic efficiency than LinB toward five of the six tested substrates (Table 1 ). Its $k_{\text {cat }}$ value and catalytic efficiency $\left(k_{\text {cat }} / K_{\mathrm{m}}\right)$ were highest $\left(1.6 \mathrm{~s}^{-1}\right.$ and 22.5 $\mathrm{M}^{-1} \mathrm{~s}^{-1}$, respectively) for the conversion of 1,3-dibromopropane. AncHLD-RLuc has higher catalytic efficiency for 1,3dibromopropane than $\operatorname{LinB}\left(k_{\mathrm{cat}} / K_{\mathrm{m}}=1.7 \mathrm{M}^{-1} \mathrm{~s}^{-1}\right)$ due to 339-fold lower $K_{\mathrm{m}}$ (Table 1$)$. The catalytic efficiencies of AncHLD-RLuc and LinB were similar for the reaction with 1bromobutane $\left(k_{\text {cat }} / K_{\mathrm{m}}=12.8\right.$ and $18.7 \mathrm{mM}^{-1} \mathrm{~s}^{-1}$, respectively). The catalytic efficiency of AncHLD-RLuc was lowest for the reactions with 1,2-dibromoethane $\left(k_{\mathrm{cat}} / K_{\mathrm{m}}=\right.$ $\left.0.17 \mathrm{mM}^{-1} \mathrm{~s}^{-1}\right)$ and 1,2,3-tribromopropane $\left(k_{\mathrm{cat}} / K_{\mathrm{m}}=0.87\right.$ $\mathrm{mM}^{-1} \mathrm{~s}^{-1}$ ), mainly due to low turnover numbers. Substrate inhibition of both AncHLD-RLuc and LinB was observed with four of the six halogenated substrates, and weak cooperativity identified with three of the six substrates (Table S3). Kinetics 
A

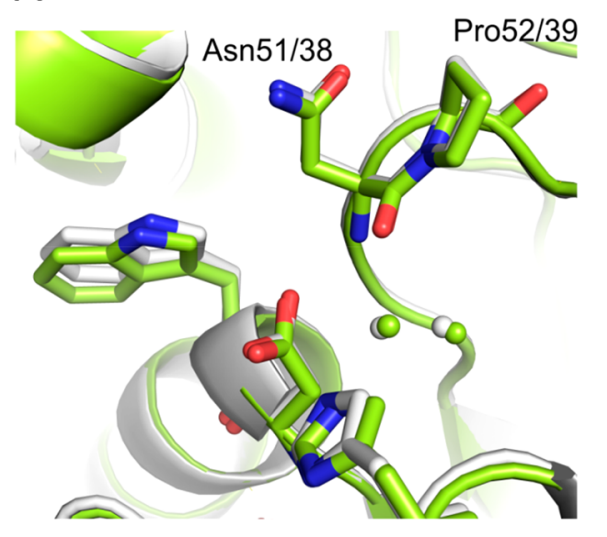

B

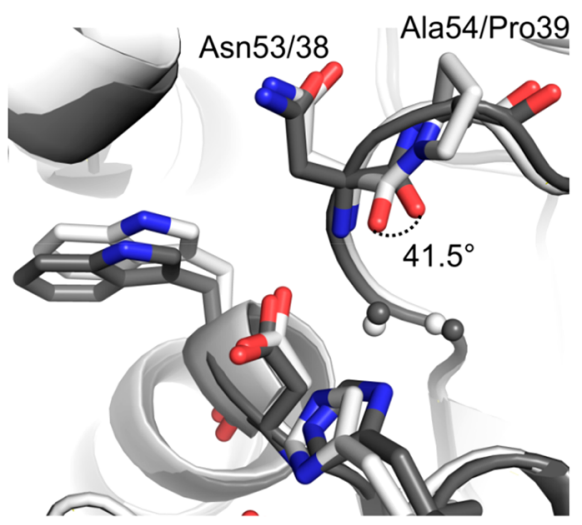

C

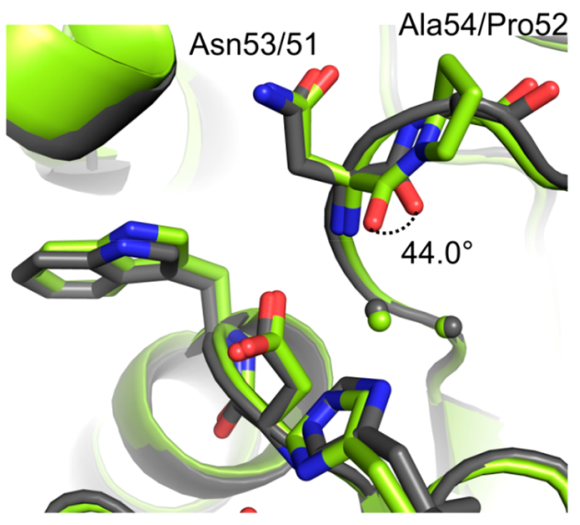

Figure 3. Molecular adaptation to distinct enzymatic reactions by a single-point mutation in the residue adjacent to the catalytic residue. Differences in spatial orientation of the backbone carbonyl group of the catalytic halide/oxygen-stabilizing residues Asn51 (AncHLD-RLuc), Asn53 (RLuc8), and Asn38 ( $\mathrm{LinB}$ ) due to different side chains of neighboring Pro/Ala residues. (A) Superimposition of the active site residues of AncHLD-RLuc and LinB. (B) Superimposition of the active-site residues of RLuc8 and LinB. (C) Superimposition of the active site residues of RLuc8 and AncHLD-RLuc. C atoms of the active site residues and water molecules of AncHLD-RLuc (green), Rluc8 (black), and LinB (white). Angles of rotation of the backbone carbonyl of Asn53 in RLuc8 relative to the orientation of corresponding carbonyl groups in LinB and AncHLDRLuc are indicated by black dashed lines.

of AncHLD-RLuc and RLuc8 with coelenterazine followed simple Michaelis-Menten dependence $\left(V_{\lim }=73\right.$ and $1.6 \times$ $10^{7} \mathrm{RLUs}^{-1} \mathrm{mg}^{-1} ; K_{\mathrm{m}}=1.3$ and $1.1 \mu \mathrm{M}$, respectively). Steadystate kinetic data obtained for the reaction with coelenterazine confirmed that AncHLD-RLuc has 6 orders of magnitude weaker monooxygenase activity than RLuc8 (Table 1).

Structural Analysis of AncHLD-RLuc. AncHLD-RLuc was crystallized and its tertiary structure was solved by X-ray crystallography to $1.39 \AA$ resolution (Table S1), with two copies of the enzyme in the asymmetric unit. The search model used for the structure determination was RLuc8 (PDB ID 2PSF). ${ }^{67}$ The final structural model contains the residues 12 307. The overall structure of AncHLD-RLuc is composed of an $\alpha / \beta$ hydrolase core domain and a helical cap domain (Figure $2 \mathrm{~A}, \mathrm{~B})$. The core domain consists of a central eight-stranded $\beta$ sheet with $\beta 2$ lying in an antiparallel orientation with respect to the $\beta$-sheet. The $\beta$-sheet is surrounded by six $\alpha$-helices. The cap domain is composed of five $\alpha$-helices. The hydrophobic cavity of AncHLD-RLuc is located between the core and the cap domain. The cavity is connected to the protein surface by two access tunnels and contains five catalytic residues: the nucleophile Asp118; the catalytic base His284; the catalytic acid Glu142; and two halide/oxygen-stabilizing residues, Asn51 and Trp119 (Figure 2A,C). This catalytic pentad is known to be essential for HLD activity ${ }^{27,30}$ and to a large extent for monooxygenase activity. ${ }^{26,29}$

During refinement of the crystal structure, strong electron density of potential ligands was detected in the vicinity of the AncHLD-RLuc active site. Three ligands were subsequently modeled in the electron density, with occupancy 1 , in both enzyme molecules within an asymmetric unit. The first ligand was interpreted as a molecular oxygen, which serves as the cosubstrate during the monooxygenation reaction (Figure 2D). Molecular oxygen occupies the typical binding site of halogen anion released during the dehalogenase reaction and interacts with $\mathrm{N}$ atoms of two halide-stabilizing residues, Asn51 $\mathrm{N}^{\delta 2}$ and $\operatorname{Trp} 119 \mathrm{~N}^{\varepsilon 1}$, at distances of 3.07 and $3.19 \AA$, respectively (Figure 2D). Further coordination is provided by a water molecule mediating contact with the side chain of the catalytic residue Asp118. The distance between the $\mathrm{O}$ atom of the water molecule and the $\mathrm{O}^{2}$ atom of the oxygen molecule is $2.95 \AA$. The position of the molecular oxygen suggests that highly conserved halide-stabilizing residues are involved in stabilization not only of the leaving halide during dehalogenation ${ }^{88,89}$ but also molecular oxygen during the monooxygenase reaction.

Because the position of molecular oxygen is typically occupied by a halide ion in a majority of known HLD structures, ${ }^{59}$ we tried to build a chloride into the identified eggshaped electron density map. Calculation of the difference $F_{o}$ $-F_{c}$ and simulated-annealing omit electron density maps revealed that the chloride ion does not fit to the density. We then tried to build one or two water molecules into the density, but again, several cycles of structural refinements and map calculations indicated that this does not lead to a correct solution. Finally, based on the calculation of $2 m F_{o}-D F_{c}$, the $F_{\mathrm{o}}-F_{\mathrm{c}}$ difference, and simulated-annealing omit electron density map, inspection of geometry, and distances to surrounding amino acids, the density was assigned to a molecular oxygen. The other two ligands, (4S)-2-methyl-2,4pentanediol and tris(hydroxymethyl)aminomethane (Figure S6), originate from storage and cryoprotectant solutions and are not relevant for enzymatic activity.

AncHLD-RLuc Differs from RLuc8 in a Geometry of Backbone Carbonyl of a Catalytic Residue. Comparison of AncHLD-RLuc (PDB ID 6G75) with RLuc8 (PDB ID $2 \mathrm{PSF})^{67}$ and $\mathrm{LinB}$ (PDB ID $\left.1 \mathrm{MJ5}\right)^{73}$ revealed that the proteins' backbones had highly similar geometry, despite the fact that amino acid differences between the predicted ancestor and both modern enzymes are spread throughout the protein structure (Figure S7). We obtained RMSD values of 0.49 and $0.43 \AA$ for the overlaps of AncHLD-RLuc's backbone atoms with those of RLuc8 and $\mathrm{LinB}$, respectively (Figure 2B). Spatial arrangements of the side chains of catalytic residues of all three enzymes are even more highly conserved, with RMSD values of 0.23 and $0.19 \AA$ for corresponding overlaps of their catalytic pentads (Figure 2C). Clear differences in the three compared structures lie in the spatial arrangements of $\alpha 4$ helices of the cap domain, lining the main access tunnel, an adjacent loop connecting the $\alpha 4$ helix of the cap domain with the $\beta 6$ strain of the $\beta$-sheet of the main domain, and a loop 
connecting the $\alpha 7$ helix of the cap domain with the $\alpha 8$ helix of the main domain (Figures 2B and S8). Both of those loops affect the shape of the slot access tunnel, and all three structural elements are important for the size and accessibility of the buried active site and the entire cap domain's dynamics.

Within the active site, an important difference was identified in the orientation of the carbonyl group of the main chain of the halide/oxygen-stabilizing residue: Asn51 in AncHLDRLuc, Asn53 in RLuc8, and Asn38 in LinB. While the spatial orientation of the backbone carbonyl group of Asn51 in AncHLD-RLuc is almost identical to that of Asn38 in LinB, the backbone carbonyl of Asn53 in RLuc8 is rotated by $41.5^{\circ}$ and $44.0^{\circ}$ relative to orientations of its analogues in $\operatorname{LinB}$ and AncHLD-RLuc (Figure 3). These differences are due to differences in side chains of residues adjacent to the halide/ oxygen-stabilizing residue in AncHLD-RLuc/LinB and RLuc8 (Pro and Ala, respectively). The cyclic pyrrolidine side chain fixes the backbone dihedral $\phi$ angle and thus severely restricts the conformational freedom of the carbonyl group of the main chain of the preceding Asn residue in AncHLD-RLuc and LinB. In contrast, the small side-chain of the Ala residue allows high degrees of freedom of the backbone of the neighboring halide/oxygen-stabilizing residue in RLuc8. Proper orientation of the carbonyl group of the halide-stabilizing Asn is crucial for HLD activity since it contributes to the positioning of catalytic water and affects its polarization through electrostatic interaction. ${ }^{90}$

AncHLD-RLuc Differs from Descendant Enzymes in Cap Domain's Conformational Dynamics. Solvent accessibility and conformational dynamics of AncHLD-RLuc, RLuc8, and LinB were experimentally accessed by HDX-MS. AncHLD-RLuc exhibited a lower level of deuteration for most of the peptides over its amino acid sequence compared to both RLuc8 and LinB in the shorter reaction time (10 s), implying the more compact structure of the enzyme at the beginning of deuteration reaction. The important differences were identified in HD exchange kinetic profiles of the peptides corresponding to the structural elements of the cap domains (Figure S9). RLuc8 exhibited the highest HD exchange kinetics in almost all structural elements forming the cap domain. The only structural region where AncHLD-RLuc exhibits higher exchange kinetics and reaches a higher level of deuteration is $\alpha 4$ helix and the adjacent loop. LinB, on the other hand, exhibited the smallest $\mathrm{HD}$ exchange kinetics from all tested enzymes.

The structural elements forming the cap domain affect the size of the main access tunnel and its accessibility for the ligands. We performed $4.8 \mu \mathrm{s}$ long $\mathrm{MD}$ simulations of AncHLD-RLuc, RLuc8, and LinB to study the overall dynamics of protein structures and to analyze changes in bottleneck radius of the main access tunnels. The most flexible regions in AncHLD-RLuc, RLuc8, and LinB identified by MD simulations match the regions exhibiting the highest HD exchange kinetics analyzed by HDX-MS. The simulations revealed that the structures can adopt open and closed states of the main access tunnel due to the conformational dynamics of the secondary elements and connecting loops, particularly the $\alpha 4$ helix and the adjacent loop connecting the cap domain with the main domain (Figure S9). The maximal tunnel opening (corresponding to maximal bottleneck radius) was observed at the highest RMSD with respect to the crystal structures. AncHLD-RLuc exhibited the maximal radius bottleneck $\sim 1.8$ $\AA$, while both descendants RLuc8 and LinB showed the maximal bottleneck radius $\sim 2.2 \AA$ (Figure 4). Strikingly, the probability that the main tunnel in $\operatorname{Lin} B$ is in its open state is

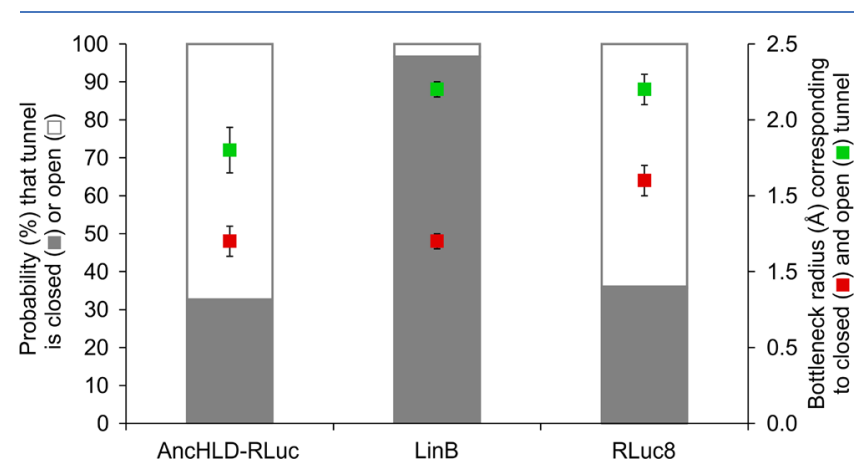

Figure 4. Conformational dynamics of the cap domain and the main access tunnel of AncHLD-RLuc, LinB, and RLuc8. MD simulations revealed that the tunnels of all three enzymes exist in open and closed states. The probability that the main tunnel is in a closed (gray) or open (white) state is represented by respective bars. The average bottleneck radius corresponding to the closed (red) and open (green) state of the main tunnel is represented by squares with the standard deviations depicted by black bars.

only $3 \%$. On the contrary, the probability that the main tunnels in AncHLD-RLuc and RLuc8 are open is 67 and 64\%, respectively (Figure 4). The tunnels found in the closed state corresponds to the lowest RMSD with respect to the crystal structures. In the closed state, AncHLD-RLuc and LinB exhibited a bottleneck radius $\sim 1.2 \AA$, while RLuc8 exhibits a significantly larger tunnel with a $\sim 1.6 \AA$ bottleneck radius.

Demonstration That a Single-Point Substitution Can Bestow HLD Activity on RLuc8. The catalytic halidestabilizing residue Asn is followed by Pro in all members of the HLD-II enzyme subfamily, while Ala in the corresponding position is unique to RLuc8. An Ala54Pro variant of RLuc8 was therefore constructed to determine whether this residue could be responsible for RLuc8's lack of HLD activity. CD spectroscopy revealed that the variant RLuc8+Ala54Pro is correctly folded and has comparable stability to RLuc8 (Figure S5). Specific activities of RLuc8+Ala54Pro were determined with 29 halogenated compounds and coelenterazine (Table S2). Strikingly, RLuc8+Ala54Pro exhibited clearly detectable dehalogenase activity toward 21 of the 29 halogenated compounds, with the preference toward terminally disubstituted brominated ethanes and propanes (Figure 5 and Table S2).

The monooxygenase activity of RLuc8+Ala54Pro with coelenterazine was 13.5 -fold lower than that of the parental enzyme RLuc8 (7.4\% of RLuc8 activity), implying that emergence of HLD activity in this mutant was accompanied by a significant drop in the native monooxygenase activity. Measurements of steady-state kinetic parameters showed that RLuc8+Ala54Pro had a $K_{\mathrm{m}}$ similar to that of AncHLD-RLuc and LinB for the reaction with 1,2-dibromoethane, but 5- and 230 -fold lower $k_{\text {cat }}$, respectively (Tables 1 and S4). Moreover, strong substrate inhibition of the variant was detected $\left(K_{\mathrm{si}}=\right.$ $2.68 \mathrm{mM}$ ), implying that its low enzymatic activity with the halogenated substrate is associated with both low turnover number and strong substrate inhibition. Interestingly, substrate inhibition was also observed in the reaction of RLuc8+Ala54Pro with coelenterazine, while RLuc8 and AncHLD-RLuc follow simple Michaelis-Menten dependence with coelenter- 


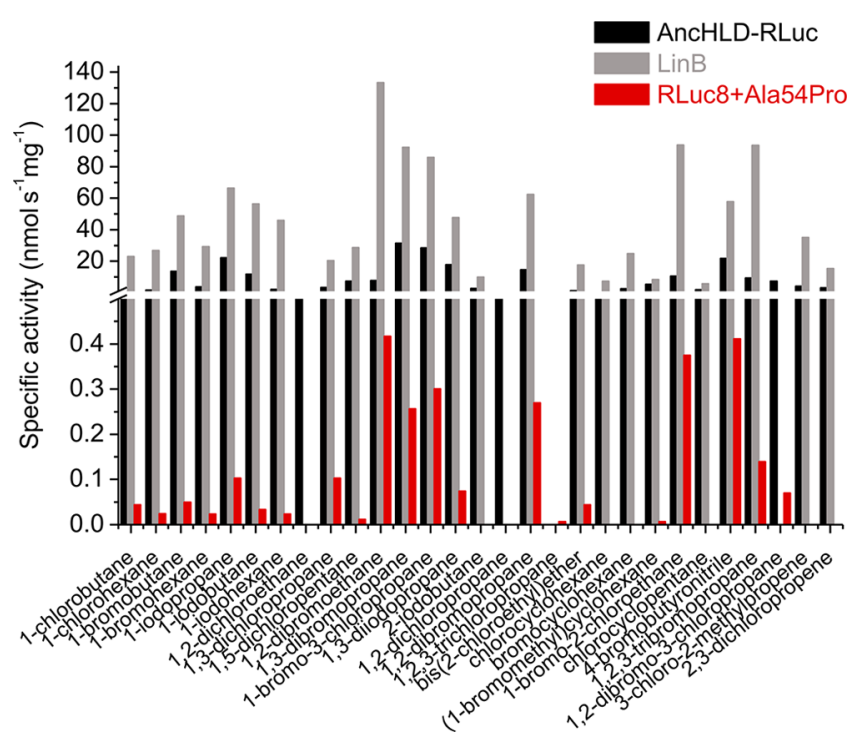

Figure 5. Comparison of the substrate specificity profile toward a set of 29 halogenated substrates of luciferase RLuc8+Ala54Pro with newly bestowed HLD activity with profiles of AncHLD-RLuc and LinB, both exhibiting efficient HLD activity. Activities were measured in three to five independent replicates with standard deviations of less than $10 \%$.

azine. In addition, the $K_{\mathrm{m}}$ of RLuc8+Ala54Pro for this substrate was 5-fold higher than that of RLuc8 and the turnover number 3-fold lower (Table S4).

Confirmation of Multiple Turnovers of RLuc8+Ala54Pro by Stopped-Flow Experiments and Mass Spectrometry. Single-turnover stopped-flow experiments with RLuc8+Ala54Pro, AncHLD-RLuc, and LinB revealed two phases during their catalytic reaction cycle with the halogenated substrate 1-bromobutane-a fast quenching phase followed by slower recovery of the enzyme fluorescence (Figures 6A and S10). These two phases observed in singleturnover fluorescence experiments correspond with a two-step hydrolytic conversion of the substrate: formation of the alkyl enzyme intermediate and halide ion via $S_{N} 2$ substitution, followed by hydrolysis of the covalent intermediate by activated water leading to formation of an alcohol product and enzyme regeneration ${ }^{39}$ (Figure 1A). Interestingly, upon mixing of RLuc8 in excess with the substrate, only a single quenching phase was observed in fluorescence traces, corresponding to formation of the intermediate (Figure 6A). This indicates that in the presence of such substrates RLuc8 forms a ternary dead-end covalent complex, via the first reaction step, which prevents turnover in the steady state. Formation of the dead-end intermediate upon interaction of RLuc8 with 1-bromobutane was further confirmed by MALDITOF MS analysis (Figure S11). Simultaneously, repeating single turnovers of 1-bromobutane and 1,2-dibromoethane catalyzed by RLuc8+Ala54Pro confirmed this variant's ability to repeatedly catalyze hydrolytic conversion of the substrate (Figures 6B and S12).

\section{DISCUSSION}

Catalytic promiscuity of enzymes has important implications for both understanding molecular evolution and protein engineering, ${ }^{91}$ but it is extremely difficult to predict. $^{92}$ Promiscuous activity of ancestral enzymes is often coupled with high stability, making them good templates for protein engineering. ${ }^{93,94}$ Enzymes' promiscuous functions may be rooted in different geometries of active sites, different binding subsites, and distinct catalytic residues or their different protonation states, and the reaction mechanisms involved may overlap or totally differ from those linked to their native functions. $^{6,92,95}$ Identification of promiscuous hydrolase and monooxygenase activities in AncHLD-RLuc supports the view that RLuc evolved from an ancestor exhibiting primarily haloalkane dehalogenase activity. Such exchanges of function between two different EC classes during the course of evolution are markedly less frequent than changes of function within the same EC class. Changing the chemistry perhaps requires several simultaneous mutations to occur, some of them leading to significant destabilization of the protein structure, or alternatively, a few amino acid residues might be candidates for changing the chemistry, whereas different substitutions could change the ligand binding. ${ }^{96}$ It is noteworthy that besides catalytically promiscuous ancestral enzymes, noncatalytic ancestors have also been recently reported as rare cases of evolutionary cross points leading to
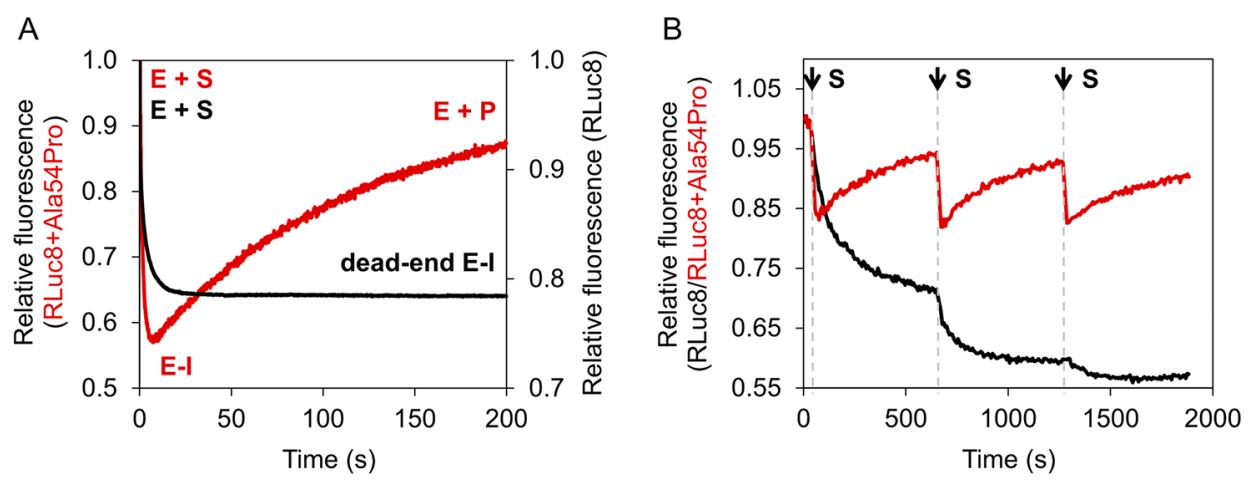

Figure 6. Results of single-turnover stopped-flow analysis of RLuc8 and RLuc8+Ala54Pro reactions with 1-bromobutane. (A) Fluorescence traces recorded following rapid mixing of $99 \mu \mathrm{M}$ RLuc8+Ala54Pro with $21.4 \mu \mathrm{M}$ of 1-bromobutane and $209 \mu \mathrm{M}$ RLuc8 with $34.5 \mu \mathrm{M} 1$-bromobutane. (B) Repetitive single-turnover stopped-flow analysis of RLuc8 and RLuc8+Ala54Pro reactions with 1-bromobutane. Fluorescence traces were recorded following mixing of $89 \mu \mathrm{M}$ RLuc8 with $85.4 \mu \mathrm{M}$ of 1-bromobutane and $137 \mu \mathrm{M}$ RLuc8+Ala54Pro with $52.7 \mu \mathrm{M}$ of 1-bromobutane, injected into the same enzyme solution in $630 \mathrm{~s}$ time intervals: E, free enzyme; S, substrate; P, product; E-I, covalently bound alkyl enzyme intermediate; dead-end E-I, ternary dead-end covalent complex that prevents enzyme turnover in the steady state. Arrows indicate the repetitive injections of the substrate. 
the emergence of catalytic activity through neofunctionalization of noncatalytic binding proteins. ${ }^{97,98}$

The ancestral enzyme AncHLD-RLuc reconstructed in this study is an efficient hydrolase that has clearly detectable monooxygenase activity. AncHLD-RLuc represents a rare case of catalytic promiscuity, as the first digit of the EC numbers of reactions it catalyzes differ. ${ }^{6}$ Such catalytic promiscuity obtained by ancestral sequence reconstruction has been previously reported for resurrected ancestors of esterases (EC 3.1.1) and hydroxynitrile lyases (EC 4.1.2), ${ }^{2,23}$ but modern esterases and lyases also reportedly have weak promiscuous lyase and esterase activity, respectively. ${ }^{22}$ The differences in reactivities of highly specific extant and promiscuous ancestral enzymes may be manifested in differences in their $k_{\text {cat }}$ and $K_{\mathrm{m}}$ values for reactions with representative substrates. Accordingly, we found that AncHLD-RLuc had a 4-fold lower $k_{\text {cat }}$ and 3-fold lower $K_{\mathrm{m}}$ than extant HLD LinB for the reaction with 1-bromobutane, resulting in very similar catalytic efficiency. Moreover, it had a similar $K_{\mathrm{m}}$ to the luciferase RLuc8 for the reaction with coelenterazine but 6 orders of magnitude lower $V_{\text {lim }}$.

Comparison of the crystal structures of the reconstructed ancestral and extant enzymes revealed high structural similarity and analogous positions of the side chains of the catalytic pentad. An important difference was discovered in the orientation of the backbone carbonyl group of the conserved Asn 53 of RLuc8, which was rotated by $41.5^{\circ}$ and $44.0^{\circ}$ relative to corresponding residues in $\mathrm{LinB}$ and AncHLD-RLuc. This rotation is due to the difference in an adjacent residue: Ala in RLuc8, instead of Pro in LinB and AncHLD-RLuc. Proper orientation of the backbone carbonyl group of the first halidestabilizing residue has known importance for positioning and polarization of catalytic water in HLDs' active sites. ${ }^{90}$ Single turnover stopped-flow experiments revealed that RLuc8 forms a covalent complex in reaction with a halogenated substrate, but it cannot catalyze its further hydrolysis. This ability was bestowed on Rluc8 by Ala54Pro mutation, which simultaneously diminished luciferase activity.

A trade-off in dual function of RLuc8+Ala54Pro is likely connected with a huge difference in size between the native substrate (coelenterazine) and the promiscuous substrate (halogenated alkane). We speculate that rigidification of the backbone carbonyl of Asn53 negatively affected the positioning of bulky coelenterazine inside the enzyme active site. Rluc8 and HLDs have different requirements for catalyzed chemical conversion. While an occluded and desolvated active site cavity with somewhat limited dynamics of access tunnels is generally beneficial for dehalogenase activity following the $S_{N} 2$ reaction mechanism, ${ }^{99,100}$ monooxygenase activity may benefit from much larger conformational flexibility, enabling binding and positioning of bulky coelenterazine. ${ }^{26,67}$

Differences in the conformational dynamics of the secondary elements forming the cap domains of AncHLD-RLuc, RLuc8, and LinB in solution were experimentally determined by HDXMS and compared with MD simulations. They are in an agreement with previously described structural requirements for dehalogenase and monooxygenase activity. Identified differences in the accessibility of the enzyme active sites connected with the probability of the main tunnel opening/ closing corroborate the necessity to modify flexibility of the secondary elements of the cap domain, in order to introduce monooxygenase activity into the dehalogenase scaffold. Rational engineering of protein conformational dynamics is an extremely challenging task and will be the focus of follow-up studies.

Detection of molecular oxygen between two halide/oxygenstabilizing residues, Asn51 and Trp119, of AncHLD-RLuc confirmed these residues' ability to stabilize halide anion produced during the HLD-catalyzed reaction ${ }^{24,88}$ as well as cosubstrate of the monooxygenase reaction. Previous sitedirected mutagenesis and molecular docking analyses indicate that these conserved residues may play important substratebinding roles in luciferase activity, particularly as hydrogenbonding partners for the $\mathrm{R}_{1}$ hydroxyl of coelenterazine. ${ }^{26} \mathrm{X}$-ray crystallography analysis performed in this study provided indications that these residues participate in the positioning of molecular oxygen in the enzyme's active site.

\section{CONCLUSIONS}

We have demonstrated that the reconstructed ancestor of evolutionary related, but catalytically distinct, HLDs and RLuc is a promiscuous enzyme with both hydrolase and monooxygenase activities. Ancestral enzyme AncHLD-RLuc exhibits a rare case of catalytic promiscuity since the EC numbers of two reactions it catalyzes differ in the first digit (3.8.1.5 versus 1.13.12.5). The crystal structure of AncHLD-RLuc solved to $1.39 \AA$ resolution revealed high structural similarity of the ancestor with both modern enzymes as well as the presence of conserved catalytic pentad. A molecular oxygen was bound in between two catalytic residues, typically involved in stabilization of a halogenated substrate and a halide ion formed during the dehalogenase reaction. This observation implies that the conserved halide-stabilizing residues participate in the coordination of molecular oxygen, playing the role of a cosubstrate during the monooxygenase reaction. Significant differences in the conformational dynamics of the secondary elements forming the specificity-determining cap domains of AncHLD-RLuc, RLuc8, and LinB were captured by HDX-MS and MD simulations. Stopped-flow and MALDI-TOF MS analyses revealed that RLuc8 lacks hydrolase activity due to its inability to hydrolyze the alkyl enzyme intermediate formed upon its interaction with halogenated substrates. A single-point substitution (Ala54Pro) adjacent to one of the halide/oxygenstabilizing residues was sufficient to bestow hydrolase activity into the RLuc8. Accumulating pieces of evidence based on this work as well as recent exciting studies ${ }^{22,23,89,90}$ suggest that ancestral sequence reconstruction has great potential for obtaining multifunctional biocatalysts.

\section{ASSOCIATED CONTENT}

\section{S Supporting Information}

The Supporting Information is available free of charge on the ACS Publications website at DOI: 10.1021/acscatal.9b01031.

Supporting tables: Specific activities and steady-state kinetic parameters of studied enzymes toward halogenated compounds and coelenterazine; diffraction data collection and refinement statistics. Supporting Figures: workflow of the study; maximum-likelihood phylogenetic tree; sequence alignment; posterior probability distribution of inferred amino acids; CD spectra and thermostability data; ligands identified in the crystal structure; structural comparison of ancestral and modern-day enzymes; expanded view of superposition of cap domains of AncHLD-RLuc, LinB, and RLuc8; HD protection plots and dynamics of AncHLD-RLuc, 
LinB, and RLuc8; single-turnover stopped-flow analysis with 1-bromobutane; MALDI-TOF MS spectra; repetitive single-turnover stopped-flow analysis with 1,2dibromoethane (PDF)

\section{AUTHOR INFORMATION}

\section{Corresponding Author}

*E-mail: jiri@chemi.muni.cz.

\section{ORCID $\odot$}

Martin Marek: 0000-0001-7220-5644

Zbynek Prokop: 0000-0001-9358-4081

Jiri Damborsky: 0000-0002-7848-8216

\section{Notes}

The authors declare no competing financial interest.

\section{ACKNOWLEDGMENTS}

The authors express sincere thanks to Professor Dan S. Tawfik (Weizmann Institute of Science, Rehovot, Israel) and Professor Uwe T. Bornscheuer (University of Greifswald, Greifswald, Germany) for extremely useful comments on the manuscript and to Irena Halikova, Jakub Kratochvil, and Hana Moskalikova (Masaryk University, Brno, Czech Republic) for technical assistance with protein preparation and characterization. The authors thank Diamond Light Source for beamtime and the staff of $\mathrm{I} 02$ beamline for technical support. This work was supported by the Grant Agency of the Czech Republic (16-24223S), the Ministry of Education, Youth, and Sports of the Czech Republic (LO1214, LQ1605, LM2015051, LM2015043, MEYS-NPS I-LO1413, and CZ.02.1.01/0.0/0.0/ 16_013/0001761), and the Ministry of Health of the Czech Republic (MH CZ-DRO MMCI, 00209805).

\section{REFERENCES}

(1) O'Brien, P. J.; Herschlag, D. Catalytic Promiscuity and the Evolution of New Enzymatic Activities. Chem. Biol. 1999, 6, R91R105.

(2) Khersonsky, O.; Roodveldt, C.; Tawfik, D. S. Enzyme Promiscuity: Evolutionary and Mechanistic Aspects. Curr. Opin. Chem. Biol. 2006, 10, 498-508.

(3) Hult, K.; Berglund, P. Enzyme Promiscuity: Mechanism and Applications. Trends Biotechnol. 2007, 25, 231-238.

(4) Humble, M. S.; Berglund, P. Biocatalytic Promiscuity. Eur. J. Org. Chem. 2011, 2011, 3391-3401.

(5) Bornscheuer, U. T.; Kazlauskas, R. J. Catalytic Promiscuity in Biocatalysis: Using Old Enzymes to Form New Bonds and Follow New Pathways. Angew. Chem., Int. Ed. 2004, 43, 6032-6040.

(6) Tawfik, D. S.; Khersonsky, O. Enzyme Promiscuity: A Mechanistic and Evolutionary Perspective. Annu. Rev. Biochem. 2010, 79, 471-505.

(7) Khersonsky, O.; Tawfik, D. S. 8.03 - Enzyme Promiscuity Evolutionary and Mechanistic Aspects. In Comprehensive Natural Products II; Elsevier: Oxford, 2010; pp 47-88.

(8) Noda-García, L.; Juárez-Vázquez, A. L.; Ávila-Arcos, M. C.; Verduzco-Castro, E. A.; Montero-Morán, G.; Gaytán, P.; CarrilloTripp, M.; Barona-Gómez, F. Insights into the Evolution of Enzyme Substrate Promiscuity after the Discovery of $(\mathrm{B} \alpha)_{8}$ Isomerase Evolutionary Intermediates from a Diverse Metagenome. BMC Evol. Biol. 2015, 15, 107.

(9) Nam, H.; Lewis, N. E.; Lerman, J. A.; Lee, D.-H.; Chang, R. L.; Kim, D.; Palsson, B. O. Network Context and Selection in the Evolution to Enzyme Specificity. Science 2012, 337, 1101-1104.

(10) Copley, S. D. Shining a Light on Enzyme Promiscuity. Curr. Opin. Struct. Biol. 2017, 47, 167-175.
(11) Newton, M. S.; Arcus, V. L.; Gerth, M. L.; Patrick, W. M. Enzyme Evolution: Innovation Is Easy, Optimization Is Complicated. Curr. Opin. Struct. Biol. 2018, 48, 110-116.

(12) Voordeckers, K.; Brown, C. A.; Vanneste, K.; van der Zande, E.; Voet, A.; Maere, S.; Verstrepen, K. J. Reconstruction of Ancestral Metabolic Enzymes Reveals Molecular Mechanisms Underlying Evolutionary Innovation through Gene Duplication. PLoS Biol. 2012, 10, e1001446.

(13) Bergthorsson, U.; Andersson, D. I.; Roth, J. R. Ohno's Dilemma: Evolution of New Genes under Continuous Selection. Proc. Natl. Acad. Sci. U. S. A. 2007, 104, 17004-17009.

(14) Thornton, J. W. Resurrecting Ancient Genes: Experimental Analysis of Extinct Molecules. Nat. Rev. Genet. 2004, 5, 366-375.

(15) Harms, M. J.; Thornton, J. W. Evolutionary Biochemistry: Revealing the Historical and Physical Causes of Protein Properties. Nat. Rev. Genet. 2013, 14, 559-571.

(16) Ogawa, T.; Shirai, T. Experimental Molecular Archeology: Reconstruction of Ancestral Mutants and Evolutionary History of Proteins as a New Approach in Protein Engineering. Protein Engineering - Technology and Application; InTech: Rijeka, Croatia, 2013; pp 111-131.

(17) Risso, V. A.; Gavira, J. A.; Mejia-Carmona, D. F.; Gaucher, E. A.; Sanchez-Ruiz, J. M. Hyperstability and Substrate Promiscuity in Laboratory Resurrections of Precambrian $\beta$-Lactamases. J. Am. Chem. Soc. 2013, 135, 2899-2902.

(18) Castro-Fernandez, V.; Bravo-Moraga, F.; Ramirez-Sarmiento, C. A.; Guixe, V. Emergence of Pyridoxal Phosphorylation through a Promiscuous Ancestor during the Evolution of Hydroxymethyl Pyrimidine Kinases. FEBS Lett. 2014, 588, 3068-3073.

(19) Huang, R.; Hippauf, F.; Rohrbeck, D.; Haustein, M.; Wenke, K.; Feike, J.; Sorrelle, N.; Piechulla, B.; Barkman, T. J. Enzyme Functional Evolution through Improved Catalysis of Ancestrally Nonpreferred Substrates. Proc. Natl. Acad. Sci. U. S. A. 2012, 109, 2966-2971.

(20) Boucher, J. I.; Jacobowitz, J. R.; Beckett, B. C.; Classen, S.; Theobald, D. L. An Atomic-Resolution View of Neofunctionalization in the Evolution of Apicomplexan Lactate Dehydrogenases. eLife 2014, 3, e02304.

(21) Steindel, P. A.; Chen, E. H.; Wirth, J. D.; Theobald, D. L. Gradual Neofunctionalization in the Convergent Evolution of Trichomonad Lactate and Malate Dehydrogenases. Protein Sci. 2016, 25, 1319-1331.

(22) Devamani, T.; Rauwerdink, A. M.; Lunzer, M.; Jones, B. J.; Mooney, J. L.; Tan, M. A. O.; Zhang, Z.-J.; Xu, J.-H.; Dean, A. M.; Kazlauskas, R. J. Catalytic Promiscuity of Ancestral Esterases and Hydroxynitrile Lyases. J. Am. Chem. Soc. 2016, 138, 1046-1056.

(23) Rauwerdink, A.; Lunzer, M.; Devamani, T.; Jones, B.; Mooney, J.; Zhang, Z.-J.; Xu, J.-H.; Kazlauskas, R. J.; Dean, A. M. Evolution of a Catalytic Mechanism. Mol. Biol. Evol. 2016, 33, 971-979.

(24) Verschueren, K. H.; Seljée, F.; Rozeboom, H. J.; Kalk, K. H.; Dijkstra, B. W. Crystallographic Analysis of the Catalytic Mechanism of Haloalkane Dehalogenase. Nature 1993, 363, 693-698.

(25) Hart, R. C.; Stempel, K. E.; Boyer, P. D.; Cormier, M. J. Mechanism of the Enzyme-Catalyzed Bioluminescent Oxidation of Coelenterate-Type Luciferin. Biochem. Biophys. Res. Commun. 1978, 81, 980-986.

(26) Fetzner, S.; Steiner, R. A. Cofactor-Independent Oxidases and Oxygenases. Appl. Microbiol. Biotechnol. 2010, 86, 791-804.

(27) Koudelakova, T.; Bidmanova, S.; Dvorak, P.; Pavelka, A.; Chaloupkova, R.; Prokop, Z.; Damborsky, J. Haloalkane Dehalogenases: Biotechnological Applications. Biotechnol. J. 2013, 8, 32-45.

(28) Lorenz, W. W.; McCann, R. O.; Longiaru, M.; Cormier, M. J. Isolation and Expression of a cDNA Encoding Renilla Reniformis Luciferase. Proc. Natl. Acad. Sci. U. S. A. 1991, 88, 4438-4442.

(29) Woo, J.; Howell, M. H.; von Arnim, A. G. Structure-Function Studies on the Active Site of the Coelenterazine-Dependent Luciferase from Renilla. Protein Sci. 2008, 17, 725-735. 
(30) Chovancova, E.; Kosinski, J.; Bujnicki, J. M.; Damborsky, J. Phylogenetic Analysis of Haloalkane Dehalogenases. Proteins: Struct., Funct., Genet. 2007, 67, 305-316.

(31) Nagata, Y.; Ohtsubo, Y.; Tsuda, M. Properties and Biotechnological Applications of Natural and Engineered Haloalkane Dehalogenases. Appl. Microbiol. Biotechnol. 2015, 99, 9865-9881.

(32) Delroisse, J.; Ullrich-Lüter, E.; Blaue, S.; Ortega-Martinez, O.; Eeckhaut, I.; Flammang, P.; Mallefet, J. A Puzzling Homology: A Brittle Star Using a Putative Cnidarian-Type Luciferase for Bioluminescence. Open Biol. 2017, 7, 160300.

(33) Ollis, D. L.; Cheah, E.; Cygler, M.; Dijkstra, B.; Frolow, F.; Franken, S. M.; Harel, M.; Remington, S. J.; Silman, I.; Schrag, J. The Alpha/Beta Hydrolase Fold. Protein Eng., Des. Sel. 1992, 5, 197-211.

(34) Nardini, M.; Dijkstra, B. W. Alpha/Beta Hydrolase Fold Enzymes: The Family Keeps Growing. Curr. Opin. Struct. Biol. 1999, 9, 732-737.

(35) Holmquist, M. Alpha/Beta-Hydrolase Fold Enzymes: Structures, Functions and Mechanisms. Curr. Protein Pept. Sci. 2000, 1, 209-235.

(36) Verschueren, K. H.; Franken, S. M.; Rozeboom, H. J.; Kalk, K. H.; Dijkstra, B. W. Refined X-Ray Structures of Haloalkane Dehalogenase at $\mathrm{PH} 6.2$ and $\mathrm{PH} 8.2$ and Implications for the Reaction Mechanism. J. Mol. Biol. 1993, 232, 856-872.

(37) Schanstra, J. P.; Ridder, I. S.; Heimeriks, G. J.; Rink, R.; Poelarends, G. J.; Kalk, K. H.; Dijkstra, B. W.; Janssen, D. B. Kinetic Characterization and X-Ray Structure of a Mutant of Haloalkane Dehalogenase with Higher Catalytic Activity and Modified Substrate Range. Biochemistry 1996, 35, 13186-13195.

(38) Bosma, T.; Pikkemaat, M. G.; Kingma, J.; Dijk, J.; Janssen, D. B. Steady-State and Pre-Steady-State Kinetic Analysis of Halopropane Conversion by a Rhodococcus Haloalkane Dehalogenase. Biochemistry 2003, 42, 8047-8053.

(39) Prokop, Z.; Monincová, M.; Chaloupková, R.; Klvaňa, M.; Nagata, Y.; Janssen, D. B.; Damborský, J. Catalytic Mechanism of the Haloalkane Dehalogenase LinB from Sphingomonas Paucimobilis UT26. J. Biol. Chem. 2003, 278, 45094-45100.

(40) Silberstein, M.; Damborsky, J.; Vajda, S. Exploring the Binding Sites of the Haloalkane Dehalogenase DhlA from Xanthobacter Autotrophicus GJ10. Biochemistry 2007, 46, 9239-9249.

(41) Babkova, P.; Sebestova, E.; Brezovsky, J.; Chaloupkova, R.; Damborsky, J. Ancestral Haloalkane Dehalogenases Show Robustness and Unique Substrate Specificity. ChemBioChem 2017, 18, 14481456.

(42) Altschul, S. F.; Madden, T. L.; Schäffer, A. A.; Zhang, J.; Zhang, Z.; Miller, W.; Lipman, D. J. Gapped BLAST and PSI-BLAST: A New Generation of Protein Database Search Programs. Nucleic Acids Res. 1997, 25, 3389-3402.

(43) NCBI Resource Coordinators. Database Resources of the National Center for Biotechnology Information. Nucleic Acids Res. 2013, 41, D8-D20.

(44) Frickey, T.; Lupas, A. CLANS: A Java Application for Visualizing Protein Families Based on Pairwise Similarity. Bioinformatics 2004, 20, 3702-3704.

(45) Li, W.; Godzik, A. Cd-Hit: A Fast Program for Clustering and Comparing Large Sets of Protein or Nucleotide Sequences. Bioinformatics 2006, 22, 1658-1659.

(46) Edgar, R. C. MUSCLE: A Multiple Sequence Alignment Method with Reduced Time and Space Complexity. BMC Bioinf. 2004, 5, 113.

(47) Hall, T. BioEdit: A User-Friendly Biological Sequence Alignment Editor and Analysis Program for Windows 95/98/NT. Nucleic Acids Symp. Ser. 1999, 41, 95-98.

(48) Guindon, S.; Dufayard, J.-F.; Lefort, V.; Anisimova, M.; Hordijk, W.; Gascuel, O. New Algorithms and Methods to Estimate Maximum-Likelihood Phylogenies: Assessing the Performance of PhyML 3.0. Syst. Biol. 2010, 59, 307-321.

(49) Le, S. Q.; Gascuel, O. An Improved General Amino Acid Replacement Matrix. Mol. Biol. Evol. 2008, 25, 1307-1320.
(50) Abascal, F.; Zardoya, R.; Posada, D. ProtTest: Selection of Best-Fit Models of Protein Evolution. Bioinformatics 2005, 21, 21042105.

(51) Guindon, S.; Gascuel, O. A Simple, Fast, and Accurate Algorithm to Estimate Large Phylogenies by Maximum Likelihood. Syst. Biol. 2003, 52, 696-704.

(52) Hordijk, W.; Gascuel, O. Improving the Efficiency of SPR Moves in Phylogenetic Tree Search Methods Based on Maximum Likelihood. Bioinformatics 2005, 21, 4338-4347.

(53) Gascuel, O. BIONJ: An Improved Version of the NJ Algorithm Based on a Simple Model of Sequence Data. Mol. Biol. Evol. 1997, 14, 685-695.

(54) Yang, Z.; Kumar, S.; Nei, M. A New Method of Inference of Ancestral Nucleotide and Amino Acid Sequences. Genetics 1995, 141, $1641-1650$.

(55) Koshi, J. M.; Goldstein, R. A. Probabilistic Reconstruction of Ancestral Protein Sequences. J. Mol. Evol. 1996, 42, 313-320.

(56) Yang, Z. PAML 4: Phylogenetic Analysis by Maximum Likelihood. Mol. Biol. Evol. 2007, 24, 1586-1591.

(57) Hanson-Smith, V.; Kolaczkowski, B.; Thornton, J. W. Robustness of Ancestral Sequence Reconstruction to Phylogenetic Uncertainty. Mol. Biol. Evol. 2010, 27, 1988-1999.

(58) Fitch, W. M. Toward Defining the Course of Evolution: Minimum Change for a Specific Tree Topology. Syst. Zool. 1971, 20 406-416.

(59) Chaloupkova, R.; Prudnikova, T.; Rezacova, P.; Prokop, Z.; Koudelakova, T.; Daniel, L.; Brezovsky, J.; Ikeda-Ohtsubo, W.; Sato, Y.; Kuty, M.; Nagata, Y.; Smatanova, I. K.; Damborsky, J. Structural and Functional Analysis of a Novel Haloalkane Dehalogenase with Two Halide-Binding Sites. Acta Crystallogr., Sect. D: Biol. Crystallogr. 2014, 70, 1884-1897.

(60) Iwasaki, I.; Utsumi, S.; Ozawa, T. New Colorimetric Determination of Chloride Using Mercuric Thiocyanate and Ferric Ion. Bull. Chem. Soc. Jpn. 1952, 25, 226-226.

(61) Battye, T. G. G.; Kontogiannis, L.; Johnson, O.; Powell, H. R.; Leslie, A. G. W. IMOSFLM: A New Graphical Interface for Diffraction-Image Processing with MOSFLM. Acta Crystallogr., Sect. D: Biol. Crystallogr. 2011, 67, 271-281.

(62) Evans, P. R.; Murshudov, G. N. How Good Are My Data and What Is the Resolution? Acta Crystallogr., Sect. D: Biol. Crystallogr. 2013, 69, 1204-1214.

(63) Matthews, B. W. Solvent Content of Protein Crystals. J. Mol. Biol. 1968, 33, 491-497.

(64) Kantardjieff, K. A.; Rupp, B. Matthews Coefficient Probabilities: Improved Estimates for Unit Cell Contents of Proteins, DNA, and Protein-Nucleic Acid Complex Crystals. Protein Sci. 2003, $12,1865-1871$.

(65) McCoy, A. J.; Grosse-Kunstleve, R. W.; Adams, P. D.; Winn, M. D.; Storoni, L. C.; Read, R. J. Phaser Crystallographic Software. J. Appl. Crystallogr. 2007, 40, 658-674.

(66) Winn, M. D.; Ballard, C. C.; Cowtan, K. D.; Dodson, E. J.; Emsley, P.; Evans, P. R.; Keegan, R. M.; Krissinel, E. B.; Leslie, A. G. W.; McCoy, A.; McNicholas, S. J.; Murshudov, G. N.; Pannu, N. S.; Potterton, E. A.; Powell, H. R.; Read, R. J.; Vagin, A.; Wilson, K. S. Overview of the CCP4 Suite and Current Developments. Acta Crystallogr., Sect. D: Biol. Crystallogr. 2011, 67, 235-242.

(67) Loening, A. M.; Fenn, T. D.; Gambhir, S. S. Crystal Structures of the Luciferase and Green Fluorescent Protein from Renilla Reniformis. J. Mol. Biol. 2007, 374, 1017-1028.

(68) Emsley, P.; Cowtan, K. Coot: Model-Building Tools for Molecular Graphics. Acta Crystallogr., Sect. D: Biol. Crystallogr. 2004, $60,2126-2132$.

(69) Murshudov, G. N.; Vagin, A. A.; Dodson, E. J. Refinement of Macromolecular Structures by the Maximum-Likelihood Method. Acta Crystallogr., Sect. D: Biol. Crystallogr. 1997, 53, 240-255.

(70) Adams, P. D.; Afonine, P. V.; Bunkóczi, G.; Chen, V. B.; Davis, I. W.; Echols, N.; Headd, J. J.; Hung, L.-W.; Kapral, G. J.; GrosseKunstleve, R. W.; McCoy, A. J.; Moriarty, N. W.; Oeffner, R.; Read, R. J.; Richardson, D. C.; Richardson, J. S.; Terwilliger, T. C.; Zwart, P. 
H. PHENIX: A Comprehensive Python-Based System for Macromolecular Structure Solution. Acta Crystallogr., Sect. D: Biol. Crystallogr. 2010, 66, 213-221.

(71) Joosten, R. P.; Long, F.; Murshudov, G. N.; Perrakis, A. The PDB_REDO Server for Macromolecular Structure Model Optimization. $\bar{I} U C r J ~ 2014,1,213-220$.

(72) PyMOL molecular graphics system, version 1.5.0.4; Schrödinger, LLC.

(73) Oakley, A. J.; Klvana, M.; Otyepka, M.; Nagata, Y.; Wilce, M. C. J.; Damborský, J. Crystal Structure of Haloalkane Dehalogenase LinB from Sphingomonas Paucimobilis UT26 at 0.95 A Resolution: Dynamics of Catalytic Residues. Biochemistry 2004, 43, 870-878.

(74) Anandakrishnan, R.; Aguilar, B.; Onufriev, A. V. H++ 3.0: Automating PK Prediction and the Preparation of Biomolecular Structures for Atomistic Molecular Modeling and Simulations. Nucleic Acids Res. 2012, 40, W537-541.

(75) Doerr, S.; Harvey, M. J.; Noé, F.; De Fabritiis, G. HTMD: High-Throughput Molecular Dynamics for Molecular Discovery. J. Chem. Theory Comput. 2016, 12, 1845-1852.

(76) Harvey, M. J.; De Fabritiis, G. An Implementation of the Smooth Particle Mesh Ewald Method on GPU Hardware. J. Chem. Theory Comput. 2009, 5, 2371-2377.

(77) Harvey, M. J.; Giupponi, G.; Fabritiis, G. D. ACEMD: Accelerating Biomolecular Dynamics in the Microsecond Time Scale. J. Chem. Theory Comput. 2009, 5, 1632-1639.

(78) Feenstra, K. A.; Hess, B.; Berendsen, H. J. C. Improving Efficiency of Large Time-Scale Molecular Dynamics Simulations of Hydrogen-Rich Systems. J. Comput. Chem. 1999, 20, 786-798.

(79) Hopkins, C. W.; Le Grand, S.; Walker, R. C.; Roitberg, A. E. Long-Time-Step Molecular Dynamics through Hydrogen Mass Repartitioning. J. Chem. Theory Comput. 2015, 11, 1864-1874.

(80) Jurcik, A.; Bednar, D.; Byska, J.; Marques, S. M.; Furmanova, K.; Daniel, L.; Kokkonen, P.; Brezovsky, J.; Strnad, O.; Stourac, J.; Pavelka, A.; Manak, M.; Damborsky, J.; Kozlikova, B. CAVER Analyst 2.0: Analysis and Visualization of Channels and Tunnels in Protein Structures and Molecular Dynamics Trajectories. Bioinformatics 2018, 34, 3586-3588.

(81) Loening, A. M.; Fenn, T. D.; Wu, A. M.; Gambhir, S. S. Consensus Guided Mutagenesis of Renilla Luciferase Yields Enhanced Stability and Light Output. Protein Eng., Des. Sel. 2006, 19, 391-400.

(82) Nagata, Y.; Hynkova, K.; Damborsky, J.; Takagi, M. Construction and Characterization of Histidine-Tagged Haloalkane Dehalogenase (LinB) of a New Substrate Class from a GammaHexachlorocyclohexane-Degrading Bacterium, Sphingomonas Paucimobilis UT26. Protein Expression Purif. 1999, 17, 299-304.

(83) Marek, J.; Vevodova, J.; Smatanova, I. K.; Nagata, Y.; Svensson, L. A.; Newman, J.; Takagi, M.; Damborsky, J. Crystal Structure of the Haloalkane Dehalogenase from Sphingomonas Paucimobilis UT26. Biochemistry 2000, 39, 14082-14086.

(84) Nagata, Y.; Nariya, T.; Ohtomo, R.; Fukuda, M.; Yano, K.; Takagi, M. Cloning and Sequencing of a Dehalogenase Gene Encoding an Enzyme with Hydrolase Activity Involved in the Degradation of Gamma-Hexachlorocyclohexane in Pseudomonas Paucimobilis. J. Bacteriol. 1993, 175, 6403-6410.

(85) Fortova, A.; Sebestova, E.; Stepankova, V.; Koudelakova, T.; Palkova, L.; Damborsky, J.; Chaloupkova, R. DspA from Strongylocentrotus Purpuratus: The First Biochemically Characterized Haloalkane Dehalogenase of Non-Microbial Origin. Biochimie 2013, 95, 2091-2096.

(86) Koudelakova, T.; Chovancova, E.; Brezovsky, J.; Monincova, M.; Fortova, A.; Jarkovsky, J.; Damborsky, J. Substrate Specificity of Haloalkane Dehalogenases. Biochem. J. 2011, 435, 345-354.

(87) Brezovsky, J.; Babkova, P.; Degtjarik, O.; Fortova, A.; Gora, A.; Iermak, I.; Rezacova, P.; Dvorak, P.; Smatanova, I. K.; Prokop, Z.; Chaloupkova, R.; Damborsky, J. Engineering a de Novo Transport Tunnel. ACS Catal. 2016, 6, 7597-7610.

(88) Verschueren, K. H.; Kingma, J.; Rozeboom, H. J.; Kalk, K. H.; Janssen, D. B.; Dijkstra, B. W. Crystallographic and Fluorescence Studies of the Interaction of Haloalkane Dehalogenase with Halide
Ions. Studies with Halide Compounds Reveal a Halide Binding Site in the Active Site. Biochemistry 1993, 32, 9031-9037.

(89) Krooshof, G. H.; Ridder, I. S.; Tepper, A. W.; Vos, G. J.; Rozeboom, H. J.; Kalk, K. H.; Dijkstra, B. W.; Janssen, D. B. Kinetic Analysis and X-Ray Structure of Haloalkane Dehalogenase with a Modified Halide-Binding Site. Biochemistry 1998, 37, 15013-15023.

(90) Otyepka, M.; Banas, P.; Magistrato, A.; Carloni, P.; Damborsky, J. Second Step of Hydrolytic Dehalogenation in Haloalkane Dehalogenase Investigated by QM/MM Methods. Proteins: Struct., Funct., Genet. 2008, 70, 707-717.

(91) Zou, T.; Risso, V. A.; Gavira, J. A.; Sanchez-Ruiz, J. M.; Ozkan, S. B. Evolution of Conformational Dynamics Determines the Conversion of a Promiscuous Generalist into a Specialist Enzyme. Mol. Biol. Evol. 2015, 32, 132-143.

(92) Nobeli, I.; Favia, A. D.; Thornton, J. M. Protein Promiscuity and Its Implications for Biotechnology. Nat. Biotechnol. 2009, 27, $157-167$.

(93) Alcalde, M. When Directed Evolution Met Ancestral Enzyme Resurrection. Microb. Biotechnol. 2017, 10, 22-24.

(94) Bloom, J. D.; Labthavikul, S. T.; Otey, C. R.; Arnold, F. H. Protein Stability Promotes Evolvability. Proc. Natl. Acad. Sci. U. S. A. 2006, 103, 5869-5874.

(95) Gupta, R. D. Recent Advances in Enzyme Promiscuity. Sustainable Chem. Processes 2016, 4, 2.

(96) Furnham, N.; Dawson, N. L.; Rahman, S. A.; Thornton, J. M.; Orengo, C. A. Large-Scale Analysis Exploring Evolution of Catalytic Machineries and Mechanisms in Enzyme Superfamilies. J. Mol. Biol. 2016, 428, 253-267.

(97) Clifton, B. E.; Kaczmarski, J. A.; Carr, P. D.; Gerth, M. L.; Tokuriki, N.; Jackson, C. J. Evolution of Cyclohexadienyl Dehydratase from an Ancestral Solute-Binding Protein. Nat. Chem. Biol. 2018, 14, $542-547$

(98) Kaltenbach, M.; Burke, J. R.; Dindo, M.; Pabis, A.; Munsberg, F. S.; Rabin, A.; Kamerlin, S. C. L.; Noel, J. P.; Tawfik, D. S. Evolution of Chalcone Isomerase from a Noncatalytic Ancestor. Nat. Chem. Biol. 2018, 14, 548-555.

(99) Janssen, D. B. Evolving Haloalkane Dehalogenases. Curr. Opin. Chem. Biol. 2004, 8, 150-159.

(100) Kaushik, S.; Marques, S. M.; Khirsariya, P.; Paruch, K.; Libichova, L.; Brezovsky, J.; Prokop, Z.; Chaloupkova, R.; Damborsky, J. Impact of the Access Tunnel Engineering on Catalysis Is Strictly Ligand-specific. FEBS J. 2018, 285, 1456-1476. 